Morales Montero, O. F., Álbarran Torres, A. J., Gutiérrez Camargo, J. J. (2021). Estudio espacio temporal de la cobertura de la tierra en el norte del río Orinoco-Venezuela (1988-2010). GeoFocus, Revista Internacional de Ciencia y Tecnología de la Información Geográfica (Artículos), 28, 95-119. http://dx.doi.org/10.21138/GF.686

\title{
ESTUDIO ESPACIO TEMPORAL DE LA COBERTURA DE LA TIERRA EN EL NORTE DEL RÍO ORINOCO-VENEZUELA (1988-2010)
}

\author{
${ }^{1}$ OTTONIEL FRANCISCO MORALES MONTERO, ${ }^{2}$ ANDERSON JAVIER ÁLBARRAN TORRES, \\ ${ }^{3}$ JONALVI JOSÉ GUTIÉRREZ CAMARGO \\ Universidad de los Andes. Facultad de Ciencias Forestales y Ambientales. Mérida-Venezuela \\ Avenida Principal Chorros de Milla. Campus Universitario Forestal. Edificio Principal. Mérida. Edo \\ Mérida-Venezuela. \\ 1'geos.otto91@gmail.com, ${ }^{2}$ aalbarran@gmail.com, ${ }^{3}$ jonalviguti@gmail.com
}

\section{RESUMEN}

En la zona norte del río Orinoco-Venezuela se identifican diversas actividades antropogénicas que degradan, modifican o eliminan la vegetación natural (deforestación, cambios de uso de la tierra, expansión de la frontera agrícola y agropecuaria, expansión urbana y suburbana). Para determinar la dinámica espacial de la zona se realizó un análisis multitemporal de la cobertura de la tierra en el periodo 1988-2010. Para este fin se generaron dos mapas de cobertura de la tierra con base a la interpretación de los mapas de vegetación a nivel nacional publicados para los años 1988 y, el más actual, 2010; por superposición utilizando los Sistemas de Información Geográfica (SIG) se generó una matriz que permitió determinar los cambios ocurridos, pudiendo observar que la cobertura de áreas intervenidas aumentó considerablemente, pasando de ocupar un $21.9 \%$ para el año 1988 a un $42.5 \%$ en el año 2010. Este cambio ocurrió en detrimento de la vegetación natural, viéndose más afectados los bosques que pasaron de ocupar un $36.8 \%$ para el año 1988 a un $22.3 \%$ para el año 2010. En base a los resultados obtenidos podemos concluir que la vegetación natural de la zona norte del río Orinoco muestra tendencias a una degradación severa e incluso a su desaparición.

Palabras clave: mapas de vegetación; actividades antropogénicas; bosques; Sistemas de Información Geográfica; análisis multitemporal. 


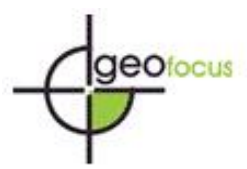

Morales Montero, O. F., Álbarran Torres, A. J., Gutiérrez Camargo, J. J. (2021). Estudio espacio temporal de la cobertura de la tierra en el norte del río Orinoco-Venezuela (1988-2010). GeoFocus, Revista Internacional de Ciencia y Tecnología de la Información Geográfica (Artículos), 28, 95-119. http://dx.doi.org/10.21138/GF.686

\title{
SPATIO-TEMPORAL STUDY FOR LAND COVER IN THE NORTH OF ORINOCO-VENEZUELA RIVER (1988-2010)
}

\begin{abstract}
In the north zone of the Orinoco-Venezuela river diverse anthropogenic activities are identified that have degraded, modified, and eliminated the natural vegetation (deforestation, changes in the land use, agricultural and farming frontier expansion, urban and suburban expansion). To determine the spatial dynamics of the zone, a multi-temporal analysis of land cover was performed in the 1988-2010 period. For this purpose, two land cover maps were generated based on the interpretation of national level vegetation maps published in years 1988 and, the most up-to-date, 2010; by crosstabulation using Geographic Information Systems (GIS) a matrix was generated, which allowed us to determine the changes that occurred, being able to observe that the coverage of intervened areas increased considerably, from $21.9 \%$ in year 1988, to $43.5 \%$ in the year 2010. This process happened in detriment of the natural vegetation of the region, forests being the most affected zones, dwindling from $36.8 \%$ in 1988 to $22.3 \%$ in 2010 . Based on these results, we conclude that the natural vegetation of the northern zone of the Orinoco River shows a tendency to severe degradation and even complete disappearance.
\end{abstract}

Key words: vegetation maps; anthropogenic activities; forests; Geographic Information Systems; multitemporal analysis.

\section{Introducción}

Sudamérica alberga el $21 \%$ de los bosques mundiales, representando aproximadamente 864000000 millones de hectáreas (FAO, 2010). El $84 \%$ del área total de bosque de la región se encuentra distribuido en cinco países, Brasil, Perú, Colombia, Bolivia y Venezuela. Dicha cubierta experimentó las mayores pérdidas netas de bosques entre el periodo 2000-2010, estimándose una pérdida de 4 millones de hectáreas anuales (FAO, 2010; FAO, 2011). Entre las principales causas de disminución de los bosques destacan la deforestación, la degradación y los cambios de cobertura de la tierra, originando la pérdida de biodiversidad e incremento de emisiones de carbono y de otros gases de efecto invernadero. La deforestación en Latinoamérica es el resultado de la expansión de la frontera agrícola representada por cultivos a gran escala y pastizales, la tala ilegal, los incendios forestales y agropecuarios, los proyectos de infraestructuras, la presión demográfica y la extracción de minerales, siendo la tasa anual de cambio estimada para la región de -1.54 (Armenteras y González, 2016; Armenteras y Rodríguez, 2014).

En este contexto, Venezuela posee una elevada diversidad vegetal que constituye un rasgo sobresaliente del espacio geográfico venezolano, donde todos los grandes biomas tropicales están representados en alguna región del país, abarcando desde las selvas pluviales hasta colonias de plantas pioneras sobre las rocas expuestas de alta montaña, así como los manglares y los páramos andinos. Es importante señalar que la distribución espacial de esta gran diversidad vegetal no se manifiesta de manera homogénea en el territorio nacional, considerando el río Orinoco como límite natural, se tiene que al norte del país se asientan los principales centros urbanos y más del $90 \%$ de la población venezolana, al sur del señalado río se encuentran alrededor de $80 \%$ de los bosques naturales y aproximadamente solo el $10 \%$ de 


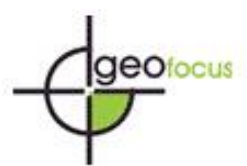

Morales Montero, O. F., Álbarran Torres, A. J., Gutiérrez Camargo, J. J. (2021). Estudio espacio temporal de la cobertura de la tierra en el norte del río Orinoco-Venezuela (1988-2010). GeoFocus, Revista Internacional de Ciencia y Tecnología de la Información Geográfica, 28, 71-95. http://dx.doi.org/10.21138/GF.686

la población. Además, las características biofísicas y socioeconómicas que influyen en la distribución espacial de la vegetación entre ambas zonas difieren ampliamente. (Huber, 2007; Instituto Geográfico Venezolano Simón Bolívar (IGVSB), 2014; Morales y Gutiérrez, 2017; Vivas, 2013).

Considerando la presión demográfica al norte del río Orinoco, los procesos de degradación, deforestación y cambios de cobertura de la tierra, se han visto reflejados en una transformación espacio temporal de los principales paisajes vegetales (Oliveira-Miranda et al., 2010), siendo la región de los Llanos uno de los paisajes más transformados, principalmente destinados a la producción agroindustrial. (Huber, 2007; Oliveira-Miranda et al., 2010).

Para observar la evolución y dinámica de un territorio, es necesario el uso de dos o más mapas de distintos años con un período de tiempo suficientemente largo que permita compararlos, y de esta manera lograr observar el cambio espacial de las coberturas de la tierra o unidades de vegetación en el tiempo. En este sentido destacan los trabajos realizados a nivel nacional de Madi et al. (2011); Morales y Gutiérrez (2017); Oliveira-Miranda et al. (2010) y Pacheco et al. (2011a), que utilizaron mapas de vegetación e imágenes satelitales para observar la dinámica espacial de la vegetación del país, logrando cuantificar los cambios, grados de intervención y ganancias y pérdidas en las categorías definidas. De manera general estas investigaciones mostraron una disminución en las coberturas vegetales del país especialmente los bosques, siendo la zona norte del Orinoco la más afectada por distintas actividades antropogénicas, principalmente la deforestación, la expansión agrícola y el crecimiento poblacional.

El objetivo central de este estudio es aplicar un análisis multitemporal para analizar los cambios de la cobertura de la tierra al norte del río Orinoco-Venezuela en el periodo 1988-2010; para ello se utilizó la cartografía de la vegetación del país realizada y publicada por Huber y Alarcón (1988), y el mapa de vegetación elaborado por Huber y Oliveira-Miranda (2010), los cuales posibilitaron la elaboración de los mapas de cobertura de la tierra que permitieron comparar las superficies por categorías y posteriormente, a partir del uso de herramientas SIG, identificar las áreas que presentaron dinamismo y las que permanecieron estables.

\section{2. Área de estudio}

El territorio continental localizado al norte del río Orinoco comprende un área de $482364.2 \mathrm{~km}^{2}$, lo que corresponde a un $52.6 \%$ del total del territorio de Venezuela, la zona norte del río Orinoco (véase figura 1) está conformada en su totalidad por las regiones naturales del sistema montañoso de la Costa, los Andes, el sistema Coriano, los Llanos, la depresión del lago de Maracaibo y gran parte de la región del delta del río Orinoco (Arismendi, 2007a; Cárdenas et al., 2000). Astronómicamente la zona está ubicada entre las coordenadas, norte: $12^{\circ} 11^{\prime} 22^{\prime} \mathrm{LN}$ y $70^{\circ} 01^{\prime}$ ' 46' ' LO en el cabo de San Román, península de Paraguaná, estado Falcón, sur: $6^{\circ} 11^{\prime} 46^{\prime}$ ' LN y $67^{\circ} 27^{\prime}$ 27' ' LO en la confluencia de los ríos Meta y Orinoco, en la intersección de los límites territoriales entre los estados Apure, Bolívar y Amazonas, este: $8^{\circ} 37^{\prime} 24^{\prime \prime}$ LN y $60^{\circ} 34^{\prime} 54$ ' LO en la desembocadura del río Grande en el sitio conocido como Boca Grande o de Navíos, estado Delta Amacuro, y oeste: $9^{\circ} 10^{\prime} 17^{\prime}$ ' LN y $73^{\circ} 25^{\prime} 00^{\prime}$ LO en el nacimiento del río Intermedio, estado Zulia, que definen sus puntos extremos. En cuanto a los límites territoriales limita al norte con el mar Caribe, y los mares territoriales de República Dominicana, de Aruba y de las Antillas Neerlandesas, de Puerto Rico e Islas Vírgenes, de Martinica y Guadalupe, y de Trinidad y Tobago; por el 


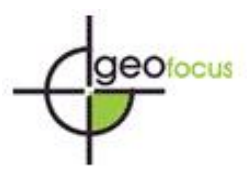

Morales Montero, O. F., Álbarran Torres, A. J., Gutiérrez Camargo, J. J. (2021). Estudio espacio temporal de la cobertura de la tierra en el norte del río Orinoco-Venezuela (1988-2010). GeoFocus, Revista Internacional de Ciencia y Tecnología de la Información Geográfica, 28, 71-95. http://dx.doi.org/10.21138/GF.686

Sur limita con la región natural del escudo de Guayana, siguiendo el curso del río Orinoco, por el Sureste limita con la región natural del delta del señalado río, por el Este con el océano Atlántico y por el Oeste y Suroeste con la República de Colombia. (Moreau, 2007).

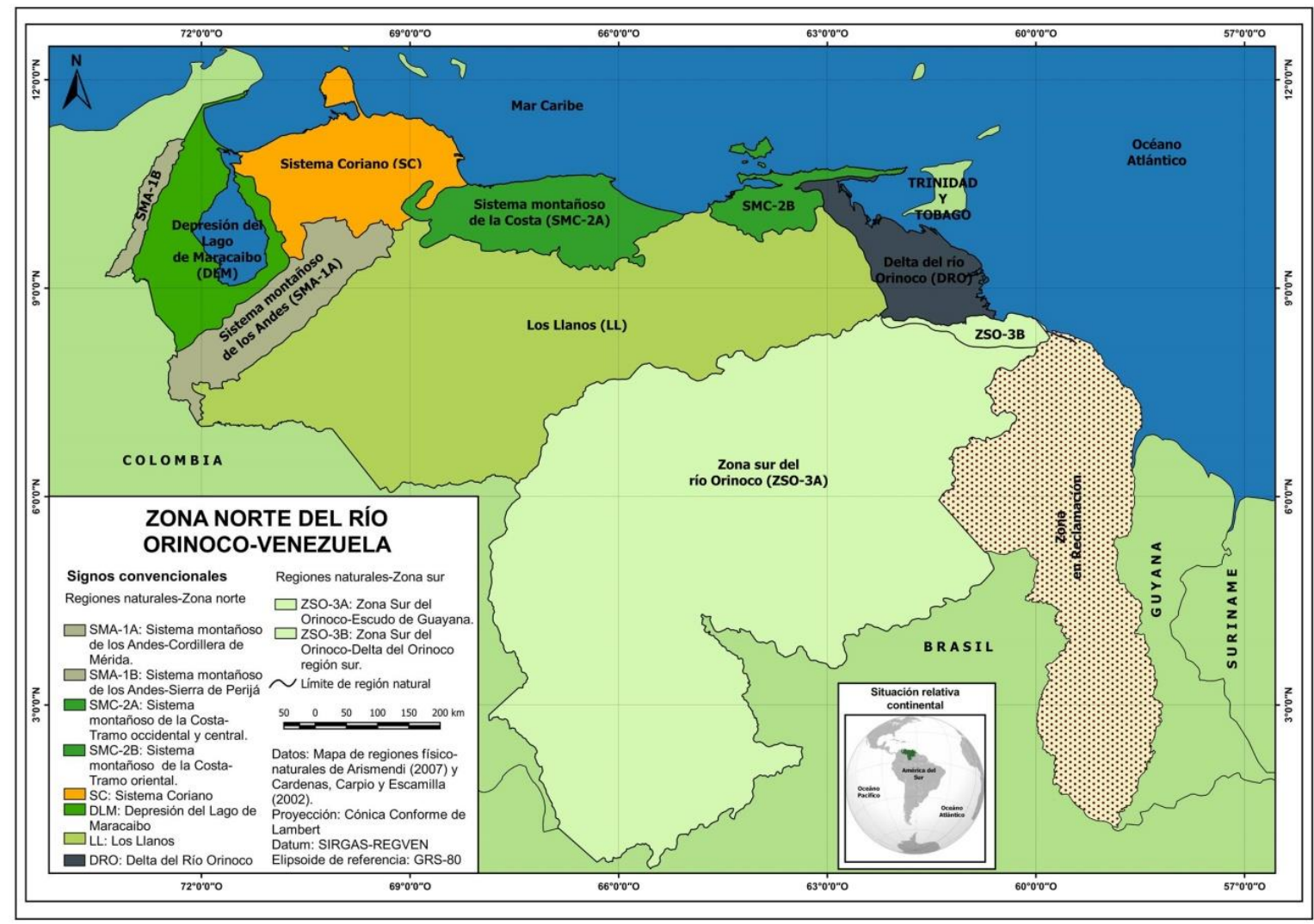

Figura 1. Localización de la zona norte del río Orinoco.

Fte: Elaboración propia con base en el mapa de unidades físico naturales de Arismendi, 2007b; Cárdenas et al., 2000

Socioeconómicamente, concentra las principales actividades económicas del país (IGVSB, 2014) las cuales se encuentran estrechamente relacionadas con las características biofísicas de las regiones naturales, y estas se encuentran bajo una alta presión antrópica ya que alberga más del $90 \%$ de la población venezolana siendo la región del sistema montañoso de la Costa donde se localizan las ciudades de mayor tamaño y son el asiento del poder político y económico del país (Morales y Gutiérrez, 2017). En cuanto a las características biofísicas, los sistemas montañosos de la zona (Sistema montañoso de los Andes, de la Costa y algunos sectores del sistema Coriano) poseen valores pluviométricos que varían entre 800 y $2800 \mathrm{~mm}$, la característica más representativa es la disminución de la temperatura con la altitud generando varios pisos térmicos y en estas regiones los suelos suelen ser de reciente evolución; Los Llanos es la segunda región natural más grande del país y a su vez la cuenca sedimentaria más extensa, su relieve plano es una de sus características más conspicua, presenta una variedad de climas cálidos y muy 


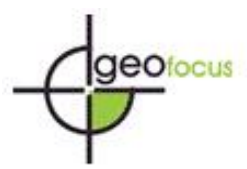

Morales Montero, O. F., Álbarran Torres, A. J., Gutiérrez Camargo, J. J. (2021). Estudio espacio temporal de la cobertura de la tierra en el norte del río Orinoco-Venezuela (1988-2010). GeoFocus, Revista Internacional de Ciencia y Tecnología de la Información Geográfica, 28, 71-95. http://dx.doi.org/10.21138/GF.686

cálidos desde húmedos hasta subhúmedos, los suelos son muy variados en cuanto a su evolución y dependen de la posición geomorfológica en que se encuentren; El sistema coriano está conformado por sierras, colinas, valles y depresiones, lo que produce una variedad de climas, desde el muy húmedo (1200 a $2400 \mathrm{~mm}$ ) hasta el árido; la región del delta del río Orinoco está determinada por la existencia de los caños de marea, los cuales se intercomunican entre sí formando islas pantanosas y grandes humedales con la existencia de un clima cálido muy húmedo y suelos recientes muy poco evolucionados; finalmente la depresión del lago de Maracaibo presenta numerosos accidentes fisiográficos como ciénagas, planicies y piedemonte además de una secuencia de subtipos climáticos que va desde el cálido superhúmedo hasta el árido. (Albarrán, 2008; Andressen, 2007; Cárdenas et al., 2000, Arismendi, 2007a).

Como resultado de la diversidad de paisajes que conforman el norte del río Orinoco, se tiene un importante mosaico de unidades de vegetación, caracterizada por: bosques en todas las regiones naturales, siendo los más representativos los bosques siempreverdes, los bosques ribereños, los bosques semideciduos y deciduos de los llanos y los bosques nublados de los Andes y la Costa; por su parte, los arbustales espinosos se concentran en la región del sistema Coriano, así como, en algunas áreas de los Andes y en varias zonas costeras asociados a climas áridos; la vegetación de páramo está restringida por las condiciones topográficas y climáticas a los Andes; los herbazales, donde se incluyen los distintos tipos de sabana se distribuyen fundamentalmente en la región de los Llanos destacando las sabanas abiertas y sabanas arbustivas y/o arboladas; los manglares se localizan en diversas áreas costeras del país siendo los más importantes en extensión los de la región del delta del Orinoco; finalmente, los matorrales se distribuyen fundamentalmente en la región del sistema Coriano y en la depresión de Unare. (Huber y Oliveira-Miranda, 2010; Huber, 2007; Huber y Alarcón, 1988).

\section{Materiales y métodos}

La reconstrucción histórica de los cambios de cobertura de la tierra requiere a menudo de la comparación de mapas de diferentes fechas y de una misma área (Petit y Lambin, 2002), siendo la técnica de detección de cambios ideal para identificar diferencias en el estado de un objeto o fenómeno sobre la superficie de la tierra mediante la observación de su evolución a lo largo de un período de tiempo, por lo tanto las diferencias espaciales observadas en las coberturas entre pares de fechas son utilizadas como indicadores para el estudio de la vegetación y del suelo, permitiendo así analizar su estado de conservación o degradación (Martínez et al., 2001). De manera general se dividió el procedimiento metodológico en 4 fases:

3.1 Búsqueda y adquisición del material cartográfico: Se realizó la búsqueda de dos productos cartográficos que mostraran la distribución espacial de la vegetación y áreas intervenidas del país, siendo estos los más adecuados para ofrecer una visión de conjunto de la diversidad de los elementos visibles en el paisaje. Dada su aceptación científica y similitud (escala, temporalidad y formaciones vegetales) se seleccionó el mapa de vegetación elaborado por Huber y Alarcón (1988), editado por el Centro Internacional de Ecología Tropical (CIET), adscrito al Instituto Venezolano de Investigaciones Científicas (IVIC), a escala 1:2000 000 (IVIC, 1998), y el mapa de formaciones vegetales de Venezuela elaborado por Huber y Oliveira-Miranda (2010) a escala 1:2 000000 disponible en el Libro Rojo de los Ecosistemas Venezolanos (Rodríguez et al., 2010), de igual manera para la delimitación 
Morales Montero, O. F., Álbarran Torres, A. J., Gutiérrez Camargo, J. J. (2021). Estudio espacio temporal de la cobertura de la tierra en el norte del río Orinoco-Venezuela (1988-2010). GeoFocus, Revista Internacional de Ciencia y Tecnología de la Información Geográfica, 28, 71-95. http://dx.doi.org/10.21138/GF.686

de las regiones naturales se utilizó el mapa de Unidades Físico Naturales de la República Bolivariana de Venezuela elaborado por Arismendi (2007b).

3.2 Homogenización del contenido temático de los mapas de vegetación: Se consideró las leyendas originales de las unidades de vegetación, el mapa del año 1988 presentaba 14 categorías y el mapa de 2010 presentaba 25, dadas las diferencias entre las unidades de vegetación entre los mapas, se realizó un proceso de generalización de las categorías base, lo cual implicó llevar ambos mapas a un mismo número de categorías que permitieron la comparación entre ellos, utilizando como criterio de homogenización la fisionomía de la vegetación, (arbórea, arbustiva, herbácea), manteniendo las categorías cuerpos de agua y áreas intervenidas (Huber y Oliveira-Miranda, 2010). Al realizar la homogenización de las leyendas temáticas, las categorías originales de ambos mapas se agruparon en 8 categorías: bosques, sabanas y herbazales, matorrales, matorrales espinosos, manglares, vegetación de páramo, cuerpos de agua y áreas intervenidas. De esta manera se llevó a cabo la salida de mapas de cobertura en un mismo número de categorías, que hizo posible su comparación.

3.3 Vectorización y elaboración de los mapas de cobertura: El mapa de vegetación de 1988 se encontraba en formato vectorial (shapefile), por lo que no fue necesario la vectorización del mismo, mientras que en el caso del mapa de vegetación para el año 2010 se procedió a la digitalización del mismo a través del método manual en pantalla utilizando el software libre SIG Qgis 2.18.19, ya que solo se encontraba en formato físico y disponible en Huber y Oliveira-Miranda (2010).

3.4 Análisis Multitemporal: Para observar los cambios espaciales de las coberturas de la tierra definidas en los mapas de cobertura, se procedió a realizar la superposición de mapas, para los años 1988 y 2010, se utilizó herramientas de análisis espacial de Qgis, y posteriormente se generó una matriz de cambio (Chuvieco, 1995; Chuvieco, 1998; Molina et al., 2009) la cual permitió identificar la direccionalidad de los cambios e indicar las áreas que se mantuvieron estables y las que presentaron dinamismo entre el período seleccionado.

\section{Resultados}

\subsection{Mapas de cobertura}

De la homogenización y reclasificación de las categorías presentes en las leyendas de los productos cartográficos originales se produjeron dos mapas de cobertura de la tierra, 1988 y 2010, figuras 2 y 3 , respectivamente. 


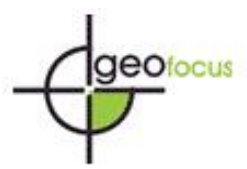

Morales Montero, O. F., Álbarran Torres, A. J., Gutiérrez Camargo, J. J. (2021). Estudio espacio temporal de la cobertura de la tierra en el norte del río Orinoco-Venezuela (1988-2010). GeoFocus, Revista Internacional de Ciencia y Tecnología de la Información Geográfica, 28, 71-95. http://dx.doi.org/10.21138/GF.686

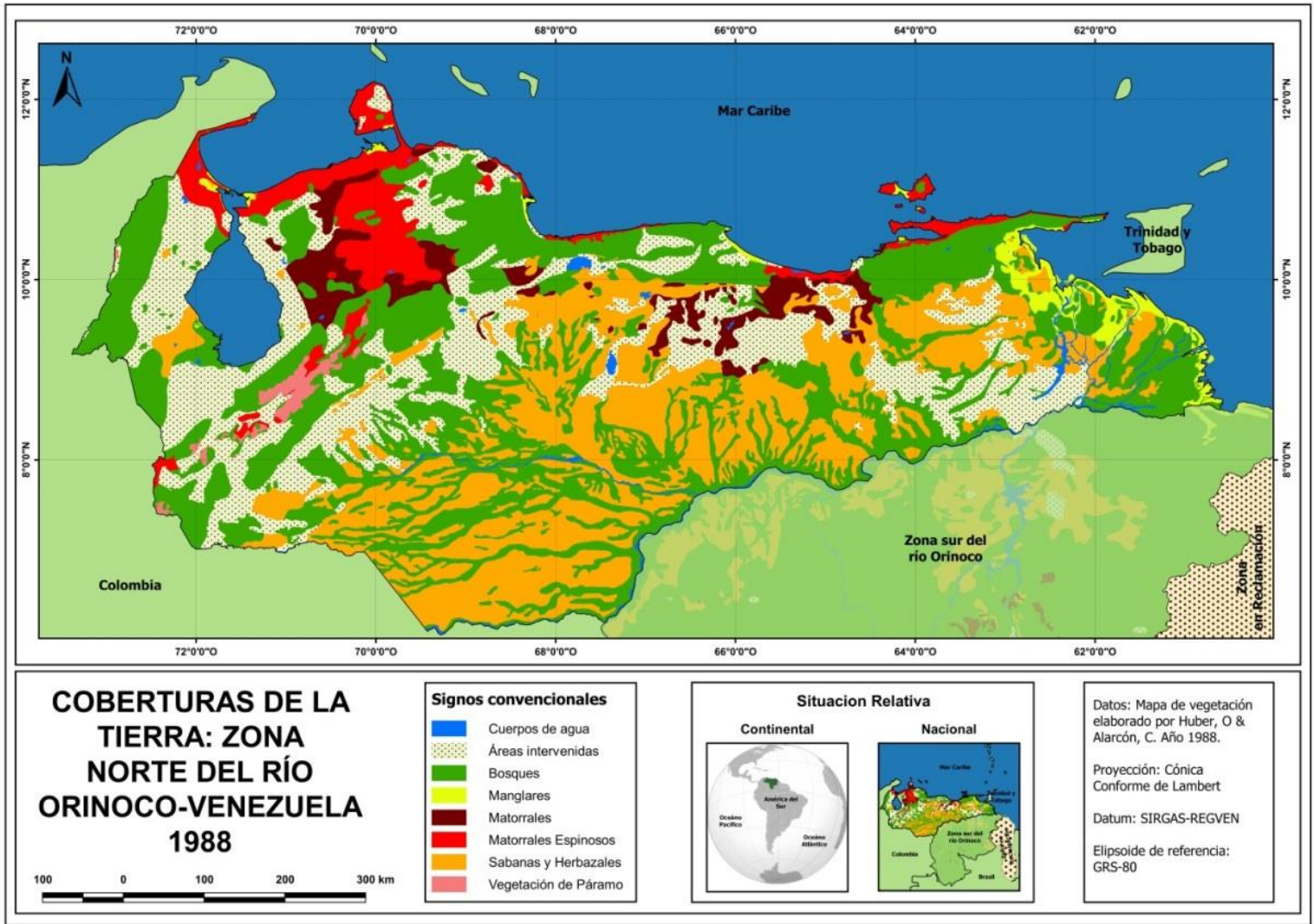

Figura 2. Mapa de cobertura de la tierra año 1988, con leyenda generalizada.

Fte: Elaboración propia con base en el mapa de vegetación de Venezuela de Huber y Alarcón. 1988.

La cobertura de la tierra que ocupó mayor superficie en porcentaje para el año 1988 es el bosque con un $36.8 \%$ del área total en estudio, en segundo lugar se encontraron las sabanas y herbazales que ocuparon un $26.6 \%$, en tercer lugar y en proporciones similares a la cobertura anterior se encuentra las áreas intervenidas que ocuparon un $21.9 \%$, siendo este el primer indicador de la fuerte intervención antropogénica a la cual ha sido sometida la zona norte del río Orinoco, en cuarto lugar se encontraron los matorrales espinosos que cubrieron un $6.3 \%$, y el resto de las categorías presentaron un porcentaje de superficie ocupada menor al $5 \%$ (véase figura 4 ). 


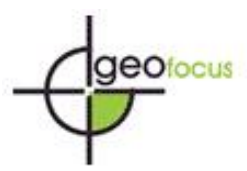

Morales Montero, O. F., Álbarran Torres, A. J., Gutiérrez Camargo, J. J. (2021). Estudio espacio temporal de la cobertura de la tierra en el norte del río Orinoco-Venezuela (1988-2010). GeoFocus, Revista Internacional de Ciencia y Tecnología de la Información Geográfica, 28, 71-95. http://dx.doi.org/10.21138/GF.686

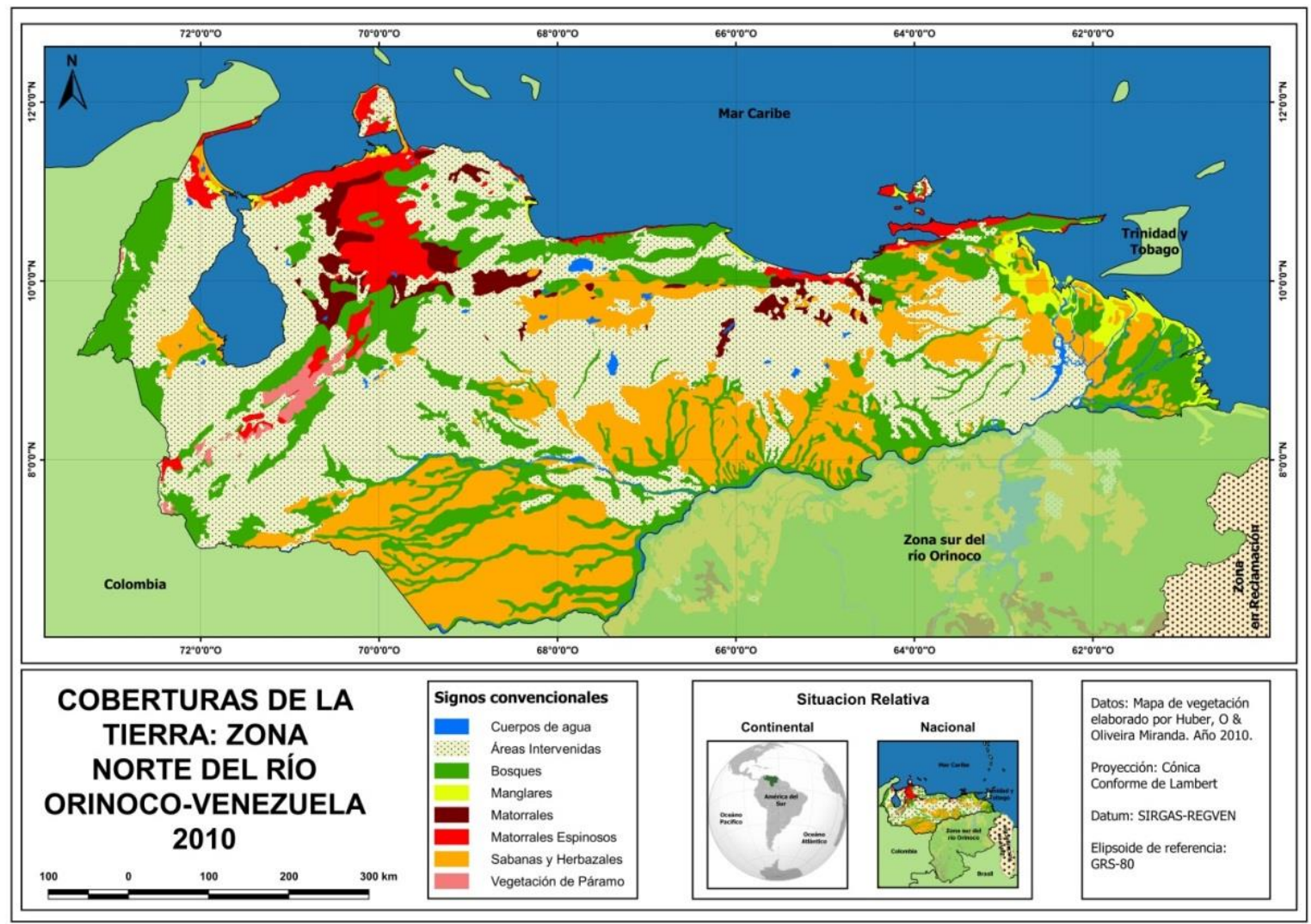

Figura 3. Mapa de cobertura de la tierra año 2010, con leyenda generalizada.

Fte: Elaboración propia con base en el mapa de formaciones vegetales de Venezuela de Huber y Oliveira Miranda. 2010.

Por su parte, la cobertura de áreas intervenidas ocupo el primer lugar en extensión de superficie para el año 2010 con un porcentaje de $43.5 \%$, siendo casi la mitad del área total en estudio, la categoría de sabanas y herbazales se encontró en segundo lugar ocupando el 22,4 \%, los bosques pasaron al tercer lugar respecto al año 1988 evidenciando una importante disminución ocupando solo un 22,3\%, los matorrales espinosos cubrieron el $5.1 \%$ y el resto de tipos de cobertura presentaron un porcentaje de ocupación de superficie menor al $5 \%$ en las cuales la mayoría presentaron una reducción de superficie con excepción de los cuerpos de agua (véase figura 5). 


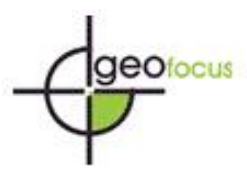

Morales Montero, O. F., Álbarran Torres, A. J., Gutiérrez Camargo, J. J. (2021). Estudio espacio temporal de la cobertura de la tierra en el norte del río Orinoco-Venezuela (1988-2010). GeoFocus, Revista Internacional de Ciencia y Tecnología de la Información Geográfica, 28, 71-95. http://dx.doi.org/10.21138/GF.686

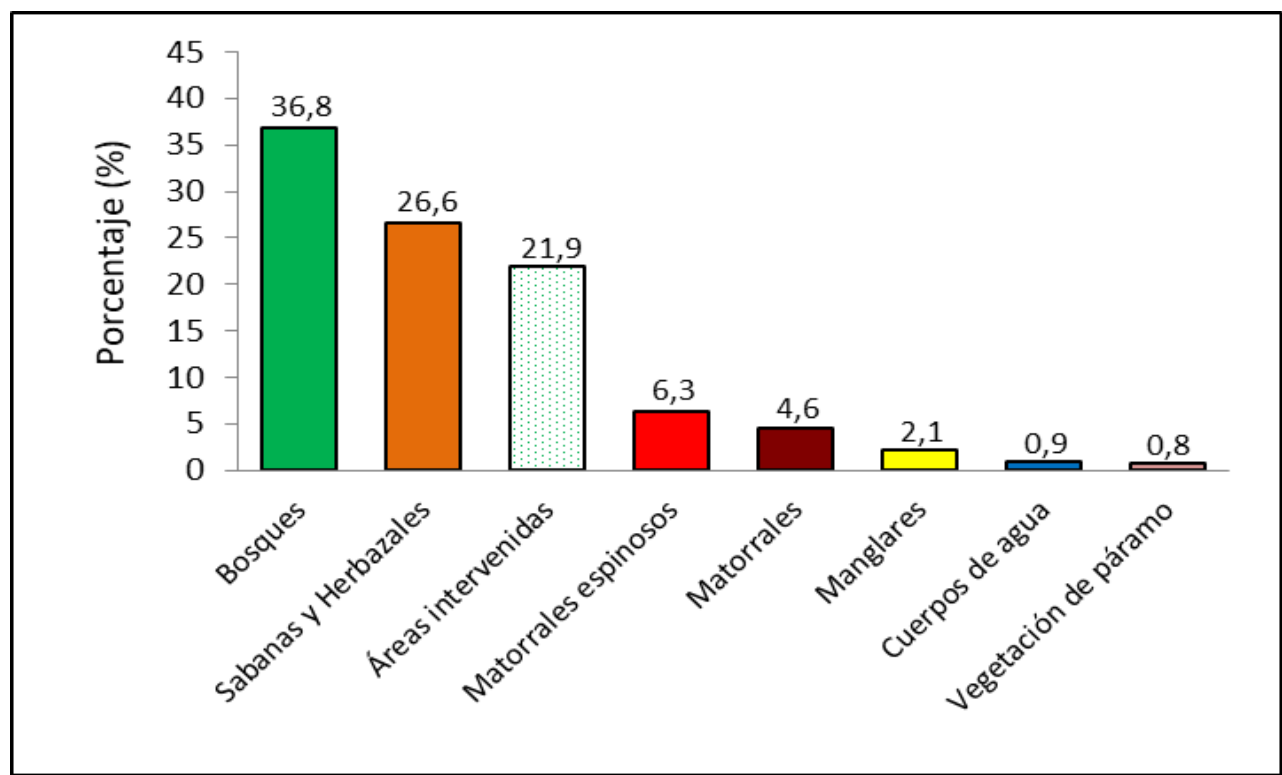

Figura 4. Superficie expresada en porcentaje (\%) por tipo de cobertura de la tierra para Venezuela. Año 1988.

Fte: Elaboración propia con base en el mapa de cobertura de la tierra de la zona norte del río Orinoco-Venezuela 1988.

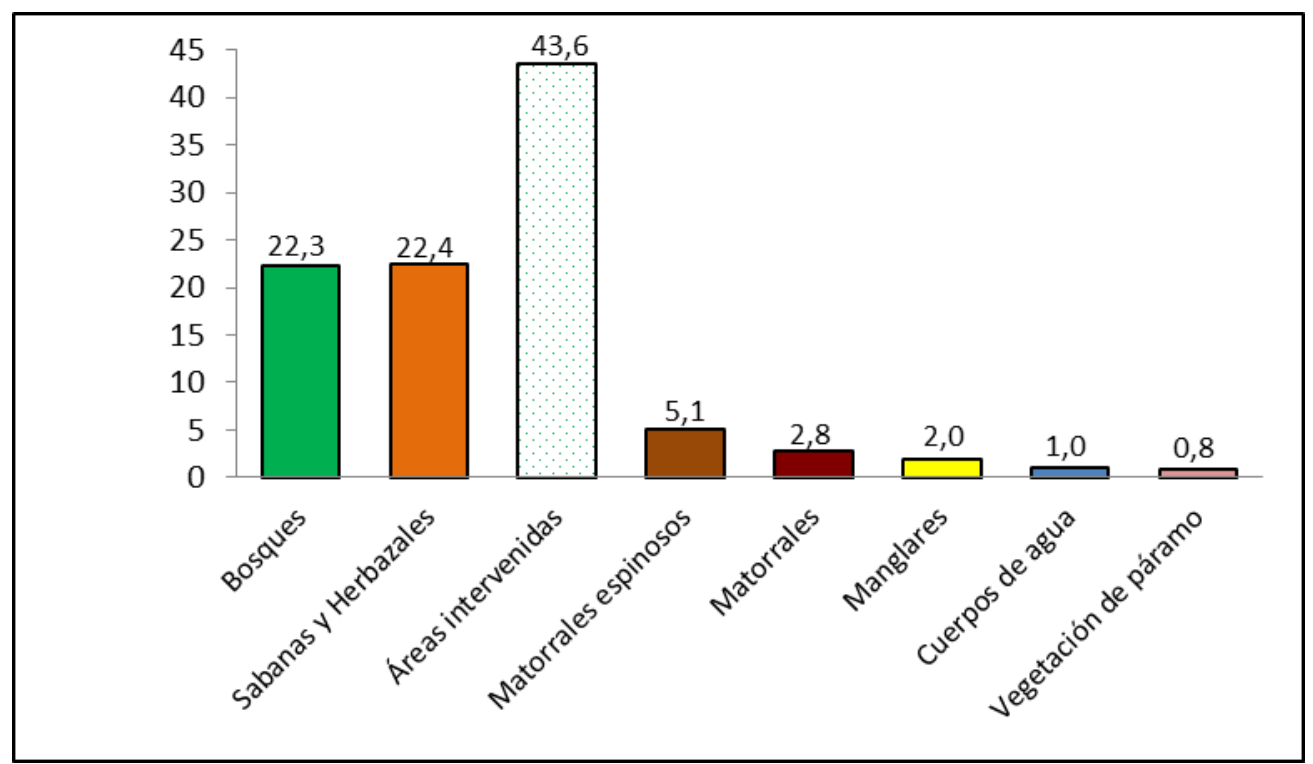

Figura 5. Superficie expresada en porcentaje (\%) por tipo de cobertura de la tierra para Venezuela. Año 2010.

Fte: Elaboración propia con base en el mapa de cobertura de la tierra de la zona norte del río Orinoco-Venezuela 2010. 


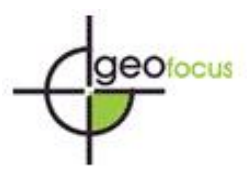

Morales Montero, O. F., Álbarran Torres, A. J., Gutiérrez Camargo, J. J. (2021). Estudio espacio temporal de la cobertura de la tierra en el norte del río Orinoco-Venezuela (1988-2010). GeoFocus, Revista Internacional de Ciencia y Tecnología de la Información Geográfica, 28, 71-95. http://dx.doi.org/10.21138/GF.686

Como se evidencia en las figuras anteriores, las áreas intervenidas aumentaron más de un $20 \%$; caso contrario ocurrió con los bosques que disminuyeron alrededor de un $15 \%$; las regiones situadas al norte del río Orinoco mostraron un alto grado de cambios, principalmente de pérdidas de vegetación natural, con un $-68,8 \%$; las mayores pérdidas de bosques se concentraron en la región Noroccidental, específicamente en los llanos occidentales y la zona sur del lago de Maracaibo, indicando la fuerte intervención a la que han estado sometidas las diversas formaciones vegetales, especialmente los bosques como consecuencia del desarrollo de actividades económicas en la zona norte del Orinoco (Pacheco et al., 2011a; Vargas y Rosales, 2014 y Bevilacqua et al., 2002).

\subsection{Comparación de superficies por categorías para el área de estudio, período 1988-2010.}

En esta fase del análisis comparativo se realizó una sustracción entre pares de categorías de los años de 1988-2010, la cual permitió observar de manera general cuales coberturas mostraron algún incremento o disminución de superficie. En la tabla 1 se muestran las coberturas que presentaron incremento en su superficie las cuales fueron: las áreas intervenidas, con un aumento de $103677 \mathrm{~km}^{2}$ y los cuerpos de agua, con un incremento de $538.6 \mathrm{~km}^{2}$.

Tabla 1. Coberturas de la tierra que presentaron aumento en su superficie, período 1988 - 2010.

\begin{tabular}{|c|r|r|r|r|r|}
\hline \multirow{2}{*}{ Categoría } & \multicolumn{2}{|c|}{2010} & \multicolumn{2}{c|}{1988} & Diferencia \\
\cline { 2 - 6 } & Área $\left(\mathrm{km}^{2}\right)$ & *Área $(\%)$ & Área $\left(\mathrm{km}^{2}\right)$ & $*$ Área $(\%)$ & Área $\left(\mathrm{km}^{2}\right)$ \\
\hline Áreas intervenidas & 209052.0 & 43.6 & 105375.0 & 21.9 & 103677.0 \\
\hline Cuerpos de agua & 4811.9 & 1.00 & 4273.3 & 0.8 & 538.6 \\
\hline
\end{tabular}

Fte: Elaboración propia con base en la superposición de los mapas de cobertura de la tierra, años 1988 y 2010. Nota: *Área \% (1988 y 2010): Superficie en \% que ocupó cada categoría con respecto a la zona norte.

Las áreas intervenidas pasaron de $105375 \mathrm{~km}^{2}$, que representaba $21.9 \%$ del área total de la zona para el año 1988, a $209052 \mathrm{~km}^{2}$, un $43.6 \%$ del área total de la zona para el año 2010, indicando que para el período de estudio la categoría se duplicó, ocupando casi la mitad de la superficie total del área. Las causas de intervención de la vegetación terrestre de Venezuela están relacionadas principalmente con los siguientes factores: la expansión agrícola y agropecuaria, la deforestación y la explotación de recursos naturales, que incluyen las actividades extractivas como la explotación forestal, petrolera y minera, las cuales han causado cambios en los usos de la tierra, para el periodo 1988-2010; las áreas intervenidas incrementaron, según Oliveira-Miranda et al. (2010), un $84 \%$ de su superficie original, concentrados estos cambios fundamentalmente en la zona norte como también lo reporta Pacheco et al. (2011b). Otro factor importante es el crecimiento urbano y la creación de nuevas infraestructuras, los cuales han ido aumentando progresivamente desde que se inició la actividad petrolera y de manera mucho más acelerada en las últimas décadas. La zona norte, según el censo realizado en el año 2011, concentraba el $93.5 \%$ de la población venezolana, la mayoría asentada en los principales centros urbanos y áreas metropolitanas de la zona (Morales y Gutiérrez, 2017). 


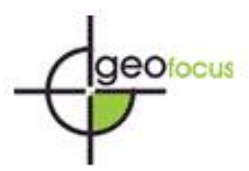

Morales Montero, O. F., Álbarran Torres, A. J., Gutiérrez Camargo, J. J. (2021). Estudio espacio temporal de la cobertura de la tierra en el norte del río Orinoco-Venezuela (1988-2010). GeoFocus, Revista Internacional de Ciencia y Tecnología de la Información Geográfica, 28, 71-95. http://dx.doi.org/10.21138/GF.686

En cuanto a los cuerpos de agua, el aumento de porcentaje corresponde a la construcción de nuevos embalses en el territorio nacional como políticas del estado para el estímulo del desarrollo agrícolas y seguridad hídrica para la zona norte del país (Grases et al., S/F)

Casi la mitad del territorio venezolano (aproximadamente el $48 \%$ ) se presentó sin intervención aparente, proporción que corresponde principalmente con la zona localizada al sur del río Orinoco, mientras que en el resto del país (principalmente al norte del Orinoco), se observó una intervención de moderada (36\%) a fuerte (41\%). En consecuencia, al menos el $77 \%$ del territorio ha sido alterado de forma significativa; la primera intervención está asociada con actividades de ganadería extensiva en hábitats de sabanas y la segunda con labores agropecuarias en zonas previamente ocupadas por diferentes tipos de bosques. Apenas el $15 \%$ del territorio localizado al norte del Orinoco presentó un buen estado de conservación de hábitats (Madi et al., 2011).

La tabla 2 muestra las coberturas que presentaron disminución en su superficie, las cuales fueron: los bosques con una disminución de $69762.3 \mathrm{~km}^{2}$, las sabanas y herbazales presentaron una pérdida de $19918.7 \mathrm{~km}^{2}$, seguidamente por los matorrales con una disminución de $8626.0 \mathrm{~km}^{2}$; posteriormente se encontraron los matorrales espinosos con $5600.2 \mathrm{~km}^{2}$, los manglares con $209.3 \mathrm{~km}^{2}$ y finalmente la vegetación de páramo con una disminución de 98.8 km².

Tabla 2. Coberturas de la tierra que presentaron disminución en su superficie para el área de estudio, período 1988 - 2010.

\begin{tabular}{|c|r|r|r|r|r|}
\hline \multirow{2}{*}{ Categoría } & \multicolumn{2}{|c|}{2010} & \multicolumn{2}{c|}{1988} & Diferencia \\
\cline { 2 - 6 } & Área $\left(\mathrm{km}^{2}\right)$ & *Área (\%) & Área $\left(\mathrm{km}^{2}\right)$ & *Área $(\%)$ & Área $\left(\mathrm{km}^{2}\right)$ \\
\hline Bosques & 107189.9 & 22.3 & 176952.2 & 36.8 & 69762.3 \\
\hline Matorrales & 13560.6 & 2.8 & 22186.7 & 4.6 & 8626.0 \\
\hline Matorrales espinosos & 24540.2 & 5.1 & 30140.4 & 6.2 & 5600.2 \\
\hline Sabanas y Herbazales & 107764.9 & 22.4 & 127683.7 & 26.5 & 19918.7 \\
\hline Vegetación de páramo & 3836.8 & 0.8 & 3935.7 & 0.8 & 98.8 \\
\hline Manglares & 9718.5 & 2.0 & 9927.9 & 2.0 & 209.3 \\
\hline
\end{tabular}

Fte: Elaboración propia con base en la superposición de los mapas de cobertura de la tierra para los años 1988 y 2010. *Área \% (1988 y 2010): Superficie en \% que ocupó cada categoría con respecto a la zona norte.

La categoría de bosques fue la que mayor superficie perdió, pasando de $176952.2 \mathrm{~km}^{2}$ para el año 1988 a $107189.9 \mathrm{~km}^{2}$ para el año 2010, significando una pérdida de bosques en la zona de $69762.3 \mathrm{~km}^{2}$. Los bosques de la zona norte se encuentran fraccionados, intervenidos y severamente degradados, provocando que cerca de dos tercios de la superficie forestal original de Venezuela al norte del Orinoco 


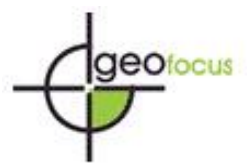

Morales Montero, O. F., Álbarran Torres, A. J., Gutiérrez Camargo, J. J. (2021). Estudio espacio temporal de la cobertura de la tierra en el norte del río Orinoco-Venezuela (1988-2010). GeoFocus, Revista Internacional de Ciencia y Tecnología de la Información Geográfica, 28, 71-95. http://dx.doi.org/10.21138/GF.686

haya sido destruida, principalmente en los llanos venezolanos, en donde los bosques húmedos, caducifolios y semicaducifolios han sido intervenidos De igual forma la mayor parte de los bosques localizados en los piedemontes de las cordilleras de Perijá, los Andes y la Costa se encuentran bajo una fuerte presión antrópica. Por otra parte, grandes áreas de bosques secos han desaparecido debido al poco consenso en integrar los ambientes modificados por la actividad humana con la conservación de la biodiversidad, y el escaso interés en utilizar planes de manejo forestal (Aymard, 2011; Aymard et al., 2011; Centeno, 2016).

Diversos factores explican esta disminución e intervención a los que han sido sometidos los bosques de la zona norte, siendo el más importante y uno de los más estudiados la deforestación. Este factor, junto a la expansión de la frontera agrícola, originan cambios en el uso de la tierra, alteración de los ecosistemas, degradación de suelos, modificación de los microclimas y pérdida de biodiversidad. Se han reportado diferentes estimaciones asociadas a la deforestación, entre ellas Plonczak (1997) indicó que la tasa de deforestación en Venezuela en la década de los 1980 ya alcanzaba una magnitud cercana a 600000 ha anuales y constituyó una de las más altas de Latinoamérica en ese momento.

Por su parte, para el 2011, Pacheco et al. (2011a) reportaron una tasa de deforestación dividida entre tres periodos con la finalidad de identificar la variación en la tasa. El primer periodo, comprendido entre 1982-1995, se estimó en $-0.93 \%$ con una pérdida de bosques de 528522 ha, mientras que para 1995-2001 se estimó una tasa aproximada de $-0.46 \%$, y para el último periodo, 2001-2008, estimaron una tasa positiva de $0.68 \%$.Esto se encuentra estrechamente relacionado con los cálculos realizados por la FAO (2011) con valores de $0.57 \%$ /año para el período de 1990-2000, 0.59\%/año para el periodo 20002005, y 0.61 para el periodo de 2005-2010, siendo la deforestación entre los periodos considerados de aproximadamente $288000 \mathrm{ha} / \mathrm{año.}$

Estos resultados situaron a Venezuela en los primeros puestos a nivel mundial en cuanto a tasas de deforestación, por causas atribuibles a: a) la expansión agrícola $(41.8 \%)$, y que se ha desarrollado principalmente en los Andes y los Llanos, b) construcción de infraestructuras (26.2\%), c) explotación de madera $(23.5 \%)$ y d) la extracción minera que representó un $5.2 \%$. Además, como causa subyacente que ha impulsado la deforestación ha sido el factor demográfico (Pacheco et al, 2011b).

Como se observa en los datos anteriores existen divergencias en las tasas de deforestación reportadas para el país; sin embargo diversas investigaciones a nivel nacional y local validan la fuerte presión antrópica sobre los bosques de la zona; entre ellas destacan los estudios elaborados por Maldonado et al. (2011), Pozzobon y Osorio (2002), Vargas et al. (2002), Aldana y Bosque (2008), Gómez y Molina (2007), León et al. (2011), Molina y Albarrán (2013), Hernández y Pozzobon (2002), Morales et al (2009), Peñaloza et al. (2008) y Pozzobon et al. (2004).

Las sabanas y herbazales fueron la segunda cobertura que más perdió superficie, pasando de $26.6 \%$ a $22.4 \%$ para el año 2010, significando una pérdida de $19918.7 \mathrm{~km}^{2}$. Las sabanas y herbazales han sido objeto de presiones antrópicas que se han incrementado desde los tiempos de la ocupación colonial, destacando la tendencia creciente a quemas inducidas que afectan la dinámica de los nutrientes en el sistema y favorecen el reemplazo de la vegetación nativa, presión de orden demográfico y económico, asociados a la explotación forestal, a la agricultura intensiva y a la ganadería extensiva, este último uno de los más importantes ya que es el más practicado en diversos paisajes de sabana especialmente en los Llanos (Oliveira-Miranda et al., 2010; José de Jesús y Montes, 2006; Silva, 2003). 
Morales Montero, O. F., Álbarran Torres, A. J., Gutiérrez Camargo, J. J. (2021). Estudio espacio temporal de la cobertura de la tierra en el norte del río Orinoco-Venezuela (1988-2010). GeoFocus, Revista Internacional de Ciencia y Tecnología de la Información Geográfica, 28, 71-95. http://dx.doi.org/10.21138/GF.686

Las siguientes coberturas perdieron superficie en el periodo de estudio pero en menor proporción que los bosques y las sabanas y herbazales: a) matorrales, b) matorrales espinosos, c) manglares y d) vegetación de páramo. Las principales causas de pérdida de superficie para cada formación vegetal señalada previamente son: a) Expansión de la frontera agrícola y de los centros poblados; b) expansión de la cría extensiva de caprinos, extracción de madera de especies como el cardón, el cují y la vera desencadenando procesos de erosión eólica e hídrica debido a la remoción de la cobertura vegetal, turismo no planificado y expansión de los asentamientos poblacionales; c) turismo intensivo, desarrollos urbanísticos no planificados, extracción de madera y leña, deforestaciones furtivas y tala, contaminación de aguas con desechos industriales, químicos, agrícolas, petroleros y urbanos; d) Expansión de la frontera agrícola y ganadería de altura, deforestación, actividades turísticas, extracción de plantas silvestres y crecimiento poblacional y urbanístico (Azocár y Fariñas, 2003; Conde y Carmona-Suarez, 2003; Matteucci y Colma, 1997, Ministerio del Ambiente y de los Recursos Naturales, 2006; Molinillo y Monasterio, 2002; Morales y Gutiérrez, 2017; Nassar et al., 2013; Oliveira-Miranda et al., 2010; Soriano y Ruiz, 2003).

\subsection{Matriz de cambio, período 1988-2010.}

Para obtener la direccionalidad de los cambios de las coberturas en el periodo de estudio se realizó la superposición entre pares de mapas. La matriz generada, expresada en $\mathrm{km}^{2}$ (véase tabla 3), señala la superficie de las coberturas que se mantuvieron estables entre pares de fechas, representadas en la diagonal de la matriz, mientras que las demás celdas muestran los cambios en el periodo seleccionado, con el fin de facilitar los análisis de la información obtenida, en la tabla 4 se muestra los valores en porcentaje (\%). Las categorías (C) definidas y señaladas en las tablas son: Áreas Intervenidas (AI), bosques (B), manglares $(\mathrm{Mg})$, matorrales $(\mathrm{M})$, matorrales espinosos $(\mathrm{Me})$, sabanas y herbazales $(\mathrm{SH})$, vegetación de páramo (VP) y cuerpos de agua (CA).

Los cambios más significativos de la cobertura de la tierra para la zona norte del río Orinoco durante el periodo 1988-2010 fueron:

Las áreas intervenidas mantuvieron una superficie invariable de $105375.0 \mathrm{~km}^{2}$, el 100\% en ambas fechas. El incremento de las áreas intervenidas está relacionado principalmente con la fuerte intervención antropogénica que ha sido ejercida en la zona, destacándose como principales causas la expansión de la frontera agrícola y agropecuaria, deforestación y el crecimiento urbano y suburbano. El incremento de esta categoría fue en detrimento de todas las formaciones vegetales presentes en la zona, principalmente por los bosques, sabanas/herbazales y matorrales.

Los bosques mantuvieron una superficie invariable de $107189.9 \mathrm{~km}^{2}$, correspondiente al $60.6 \%$ del total de superficie de esta categoría, siendo una de las más afectadas para la zona entre el periodo de estudio. Así, se tiene que el restante $39.4 \%$ de superficie para el año 2010 experimentó cambios a: Áreas intervenidas con $48205.6 \mathrm{~km}^{2}$, que representa un $27.2 \%$; matorrales con $1991.3 \mathrm{~km}^{2}$, lo que represento un $1.1 \%$; sabanas y herbazales con $19306.8 \mathrm{~km}^{2}$, lo que represento un $10.9 \%$; y a cuerpos de agua con $258.6 \mathrm{~km}^{2}$, que correspondió al $0.2 \%$. El principal cambio se da hacia la categoría de áreas intervenidas, siendo los bosques la que más aportó superficie a su favor, indicativo de la fuerte presión por parte de las actividades humanas tales como deforestación para la expansión agrícola y agropecuaria, crecimiento 


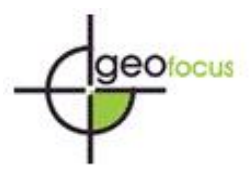

Morales Montero, O. F., Álbarran Torres, A. J., Gutiérrez Camargo, J. J. (2021). Estudio espacio temporal de la cobertura de la tierra en el norte del río Orinoco-Venezuela (1988-2010). GeoFocus, Revista Internacional de Ciencia y Tecnología de la Información Geográfica, 28, 71-95. http://dx.doi.org/10.21138/GF.686

urbano y extracción de madera, en segundo lugar, se da el cambio de bosques a sabanas y herbazales, causado por los factores señalados.

Las sabanas y herbazales mantuvieron una superficie invariable de $86940.2 \mathrm{~km}^{2}$, correspondiente al $68.1 \%$ del total de la categoría, siendo al igual que los bosques y los matorrales una de las categorías más afectadas para la zona en el periodo de estudio. El restante $31.9 \%$ de la superficie experimentó cambios hacia a: Áreas intervenidas con $40600.8 \mathrm{~km}^{2}$, lo que corresponde un $31.8 \%$; y a cuerpos de agua con $142.7 \mathrm{~km}^{2}$, lo que representó un $0.1 \%$. Las sabanas y herbazales es la segunda categoría en aportar mayor superficie hacia las áreas intervenidas, después de los bosques, causado fundamentalmente por la expansión de ganadería extensiva, agricultura intensiva, actividades agropecuarias y actividades forestales.

Los matorrales mantuvieron una superficie invariable de $11569.3 \mathrm{~km}^{2}$ correspondiendo solo al $52.2 \%$ del total de la categoría, siendo ésta la que más ha perdido su cobertura original para la zona. Las áreas de cambio se dan casi en su totalidad hacia áreas intervenidas y en una pequeña proporción hacia cuerpos de agua.

Tabla 3. Matriz de cambio de la cobertura de la tierra para la zona localizada al norte del río Orinoco-Venezuela $\left(\mathrm{km}^{2}\right)$. Período 1988 - 2010.

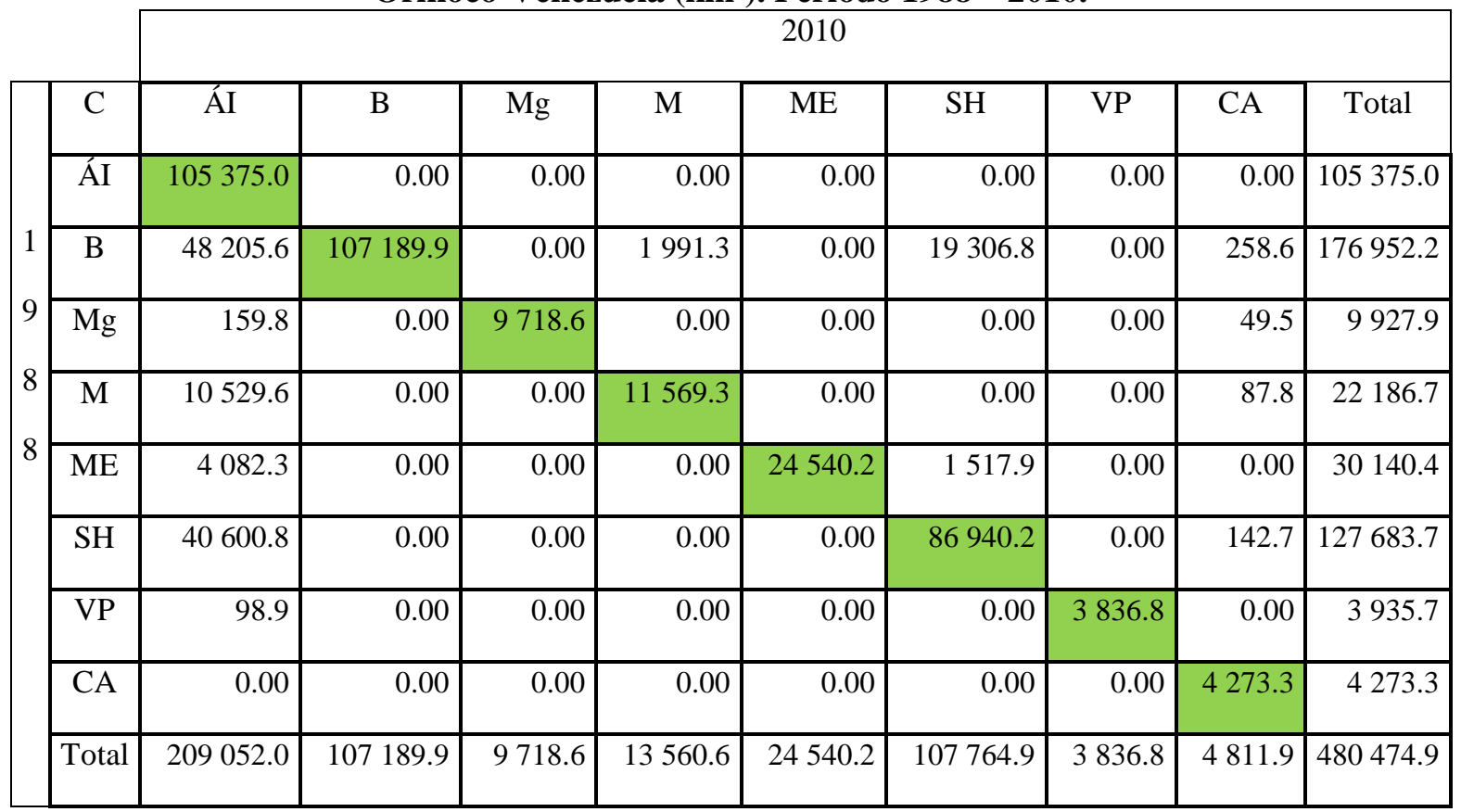

Fte: Elaboración propia con base en la superposición de los mapas de cobertura de la tierra de la zona localizada al norte del río Orinoco para los años 1988 y 2010. 


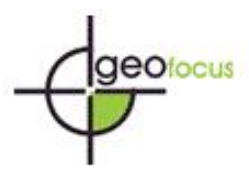

Morales Montero, O. F., Álbarran Torres, A. J., Gutiérrez Camargo, J. J. (2021). Estudio espacio temporal de la cobertura de la tierra en el norte del río Orinoco-Venezuela (1988-2010). GeoFocus, Revista Internacional de Ciencia y Tecnología de la Información Geográfica, 28, 71-95. http://dx.doi.org/10.21138/GF.686

Tabla 4. Matriz de cambio de la cobertura de la tierra para la zona localizada al norte del río Orinoco-Venezuela (\%). Período 1988 - 2010.

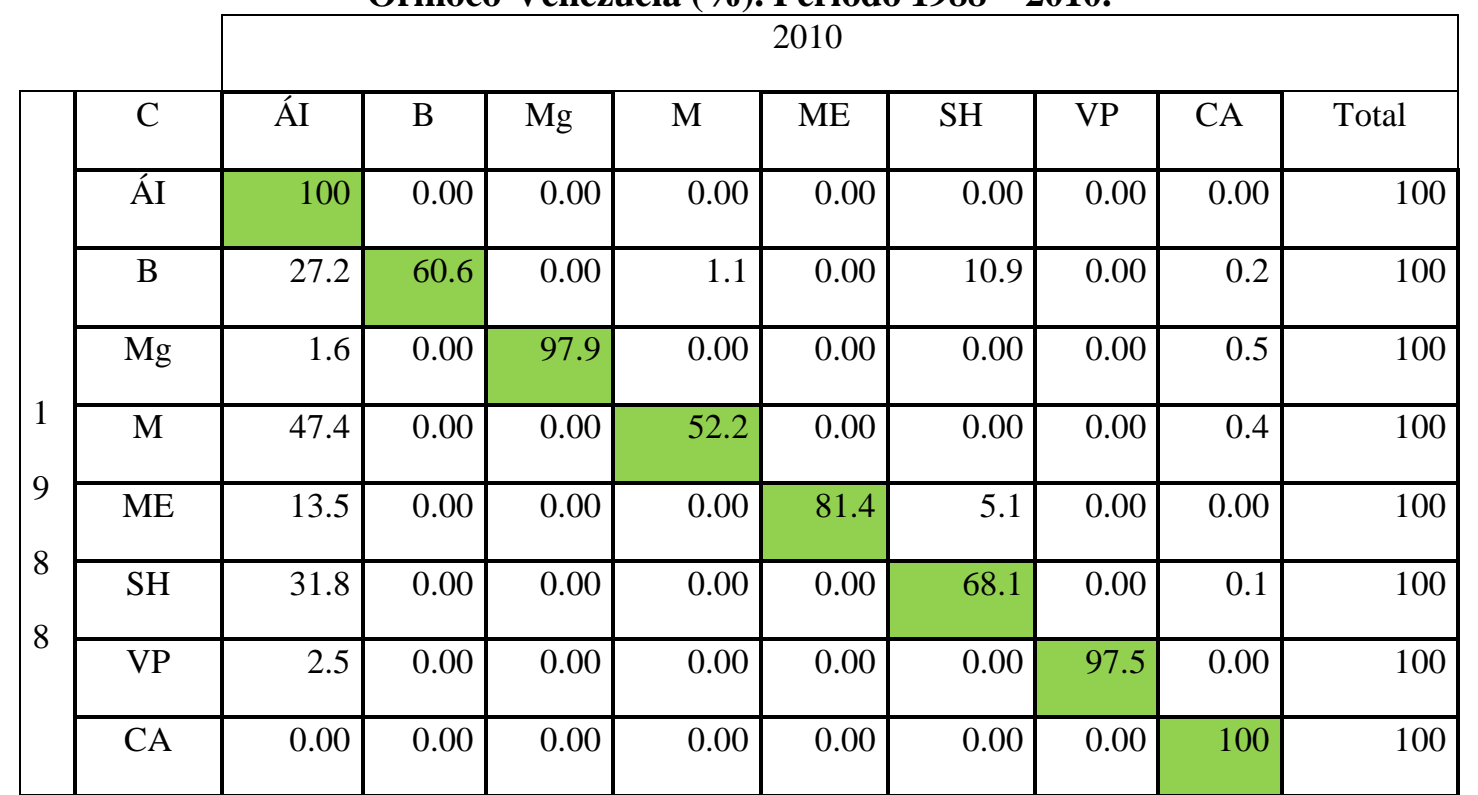

Fte: Elaboración propia con base en la superposición de los mapas de cobertura de la tierra de la zona localizada al norte del río Orinoco para los años 1988 y 2010

Los matorrales espinosos mantuvieron una superficie invariable de $24540.2 \mathrm{~km}^{2}$, manteniéndose sin cambios el $81.4 \%$ del total de la categoría. Las áreas de cambio fueron sustituidas en primer lugar por áreas intervenidas y en segundo lugar por sabanas y herbazales.

Los manglares mantuvieron una superficie invariable de $9718.6 \mathrm{~km}^{2}$, manteniéndose sin cambios un $97.9 \%$ del total de la categoría. Las pocas áreas de cambio se dan hacia áreas intervenidas y cuerpos de agua. Esta categoría es la menos intervenida de la zona ya que gran parte de éstas se sitúan en áreas donde las condiciones bio-físicas permiten que esta formación se mantenga estable, localizándose extensas áreas en las Áreas Bajo Régimen de Administración Especial (ABRAE), las cuales son diferentes figuras de protección bajo uso normado que buscan la conservación de la biodiversidad y el uso sostenible de los recursos naturales, lo que le confiere un valor adicional a la estabilidad que presenta la cobertura en el periodo analizado.

La vegetación de páramo mantuvo una superficie invariable de $3836.8 \mathrm{~km}^{2}$, correspondiente al $97.5 \%$ del total de la categoría. La poca superficie de cambio fue sustituida en su totalidad hacia las áreas intervenidas. Esta categoría es una de las menos intervenidas en la zona ya que esta cobertura se restringe a zonas de alta montaña, donde el relieve abrupto, clima riguroso y suelos con distintas limitantes influyen de manera determinante en el desarrollo de actividades humanas; adicionalmente, la mayoría de esta cobertura se encuentra bajo figuras de protección (ABRAE). 


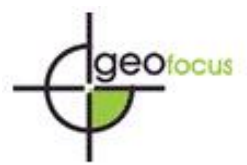

Morales Montero, O. F., Álbarran Torres, A. J., Gutiérrez Camargo, J. J. (2021). Estudio espacio temporal de la cobertura de la tierra en el norte del río Orinoco-Venezuela (1988-2010). GeoFocus, Revista Internacional de Ciencia y Tecnología de la Información Geográfica, 28, 71-95. http://dx.doi.org/10.21138/GF.686

\subsection{Mapa de cambios y permanencias-periodo 1988-2010}

En la figura 6 se muestra el mapa de cambios y permanencias con énfasis en las regiones naturales de la zona norte. Durante el periodo 1988-2010, se obtuvo que $354904.39 \mathrm{~km}^{2}$ no experimentaron cambios, lo que representó un $73.5 \%$, mientras que la superficie restante que equivale a $127459.8 \mathrm{~km}^{2}$, lo cual represento $26.4 \%$ del total de la superficie de la zona, ocurrieron cambios en las coberturas. En la figura 7 se aprecian los valores en porcentaje de las áreas de cambio y permanencia para cada región natural.

Por su parte en la tabla 5 se muestra la superficie que ocuparon las áreas de cambios y permanencias expresadas en $\mathrm{km}^{2}$ y en porcentaje para cada región para el periodo de estudio. Destacó como la región que mantuvo mayor invariabilidad espacial el delta del Orinoco, con más del $85 \%$ de áreas estables, indicando el buen estado de la vegetación que se localizó en señalada área, las condiciones bio-físicas que presenta, donde destacan selvas de difícil acceso, llanuras que se mantienen permanentemente inundados, suelos que no presentan un alto valor agrícola e importantes figuras de protección como son las ABRAE, han sido factores importantes para la preservación de la vegetación de esta región.

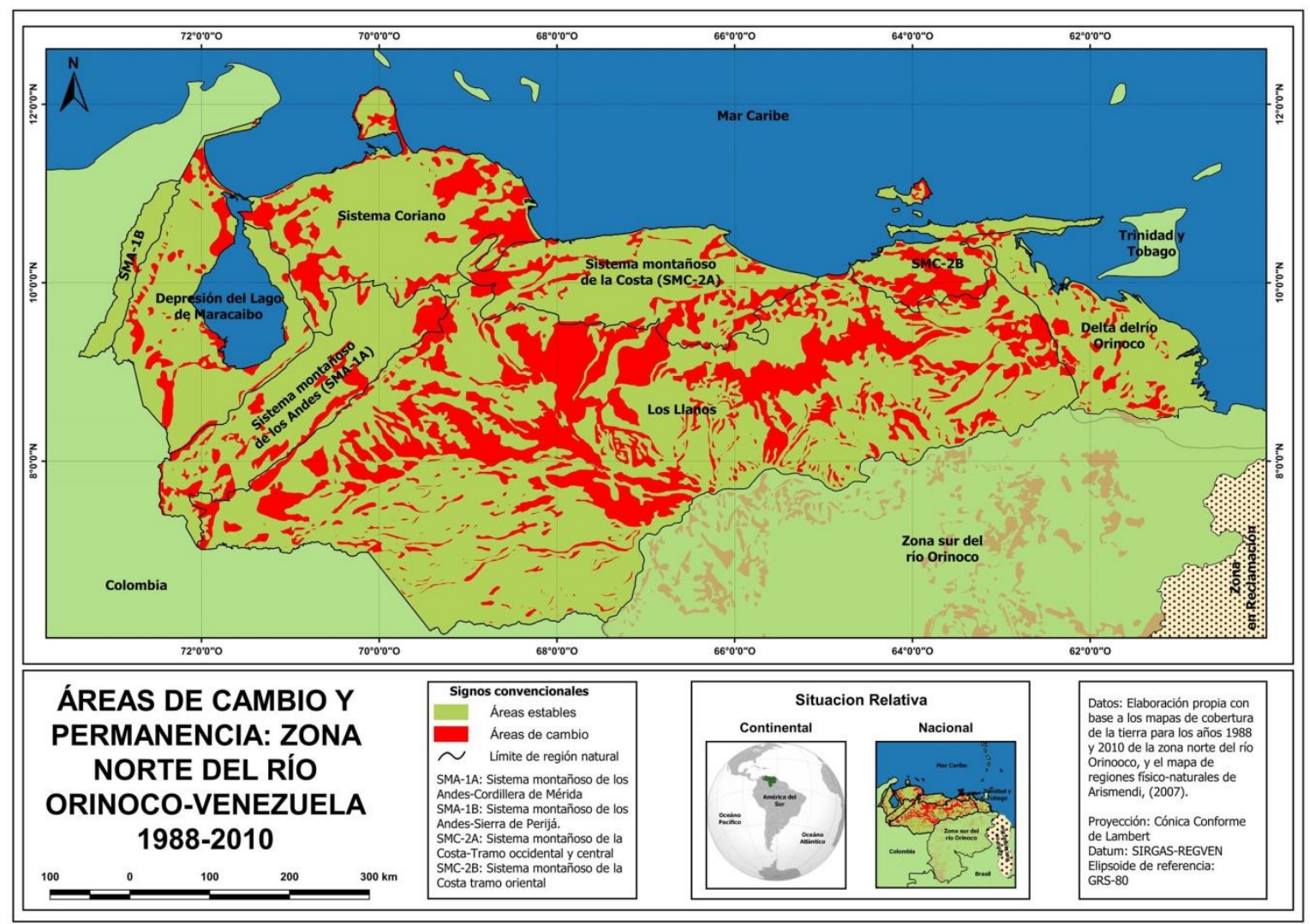

Figura 6. Mapa de áreas de cambio y permanencias de Venezuela. 1988- 2010.

Fte: Elaboración propia con base en los mapas de cobertura de la Tierra para la zona localizada al norte del río Orinoco para los años 1988 y 2010 y mapa de regiones físico naturales de Arismendi (2007b). 
Morales Montero, O. F., Álbarran Torres, A. J., Gutiérrez Camargo, J. J. (2021). Estudio espacio temporal de la cobertura de la tierra en el norte del río Orinoco-Venezuela (1988-2010). GeoFocus, Revista Internacional de Ciencia y Tecnología de la Información Geográfica, 28, 71-95. http://dx.doi.org/10.21138/GF.686

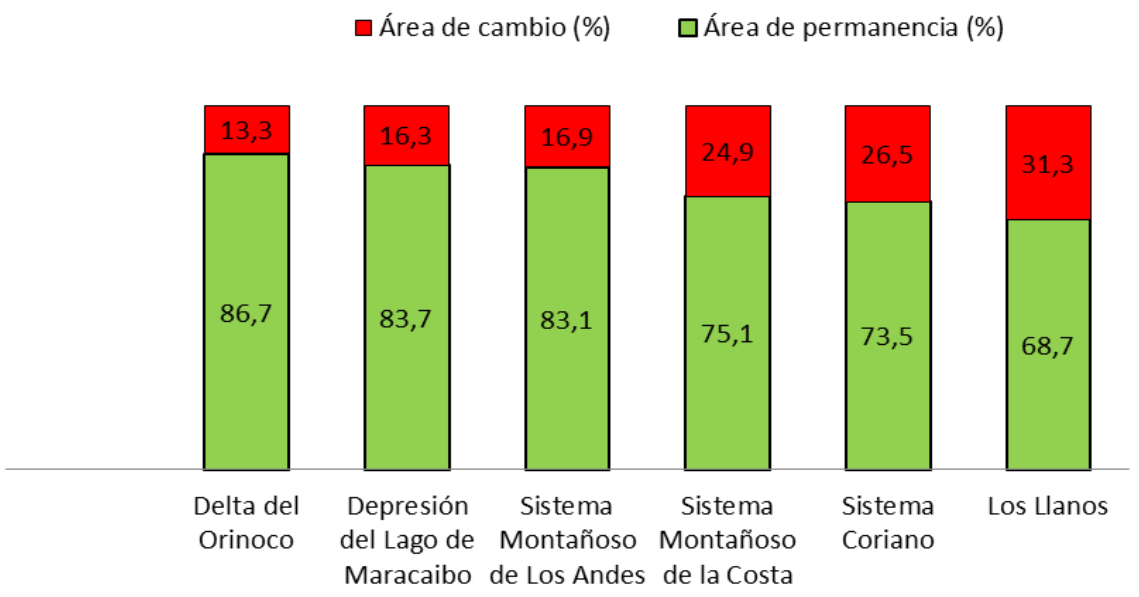

Figura 7. Áreas de cambio y permanencias expresadas en porcentaje para cada región natural de la zona norte periodo 1988-2010.

Fte: Elaboración propia con base en el mapa de cambios y permanencias.

Tabla 5. Matriz de cambio de la cobertura de la tierra para la zona localizada al norte del río Orinoco-Venezuela (\%). Período 1988 - 2010.

\begin{tabular}{|c|r|r|r|r|r|r|}
\hline Región natural & \multicolumn{1}{c|}{$\begin{array}{c}\text { Área } \\
\left(\mathbf{k m}^{2}\right)\end{array}$} & $\begin{array}{c}\text { Área } \\
(\mathbf{\%})\end{array}$ & $\begin{array}{c}\text { Área de } \\
\text { permanencia } \\
\left(\mathbf{k m}^{2}\right)\end{array}$ & $\begin{array}{c}\text { Área de } \\
\text { permanencia } \\
(\mathbf{\%})\end{array}$ & $\begin{array}{c}\text { Área de } \\
\mathbf{c a m b i o} \\
\left(\mathbf{k m}^{2}\right)\end{array}$ & $\begin{array}{c}\text { Área de } \\
\mathbf{c a m b i o} \\
(\mathbf{\%})\end{array}$ \\
\hline Delta del Orinoco & 29417.8 & 6.1 & 25514.1 & 86.7 & 3903.7 & 13.3 \\
\hline $\begin{array}{c}\text { Depresión del Lago de } \\
\text { Maracaibo }\end{array}$ & 40624.6 & 8.4 & 33990.6 & 83.7 & 6634.0 & 16.3 \\
\hline $\begin{array}{c}\text { Sistema Montañoso de Los } \\
\text { Andes }\end{array}$ & 42640.8 & 8.8 & 35421.7 & 83.1 & 7219.1 & 16.9 \\
\hline $\begin{array}{c}\text { Sistema Montañoso de la } \\
\text { Costa }\end{array}$ & 58953.5 & 12.2 & 44285.9 & 75.1 & 14667.6 & 24.9 \\
\hline Sistema Coriano & 54554.6 & 11.3 & 40092.2 & 73.5 & 14462.4 & 26.5 \\
\hline Los Llanos & 256172.5 & 53.2 & 175913.6 & 68.7 & 80258.8 & 31.3 \\
\hline
\end{tabular}

Fte: Elaboración propia con base a los datos obtenidos del mapa de cambios y permanencias.

Por otra parte, en el sistema de la Costa, sistema de los Andes, sistema Coriano, los Llanos y la depresión del lago de Maracaibo presentaron menos de $85 \%$ de áreas estables, estas regiones han sido sometidas a las diferentes actividades antropogénicas señaladas previamente, hasta el punto de que la 


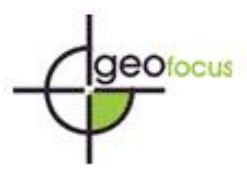

Morales Montero, O. F., Álbarran Torres, A. J., Gutiérrez Camargo, J. J. (2021). Estudio espacio temporal de la cobertura de la tierra en el norte del río Orinoco-Venezuela (1988-2010). GeoFocus, Revista Internacional de Ciencia y Tecnología de la Información Geográfica, 28, 71-95. http://dx.doi.org/10.21138/GF.686

vegetación original ha sido removida totalmente por áreas intervenidas o por otros tipos de vegetación, siendo la región de los Llanos la más afectada, presentando solo el $68.6 \%$ de áreas estables; en este proceso ha sido un factor muy importante la topografía plana de la región, lo cual permitió en gran medida el desarrollo de actividades humanas tales como la agricultura y la ganadería, esta última, fundamentalmente extensiva, además de construcción de una importante infraestructura vial que ha permitido el desarrollo económico de la región y es el eje de conexión entre las diferentes regiones del país.

Se debe considerar que la región de la depresión del lago de Maracaibo ha sido una de las más intervenidas en la zona, aunque no se ve reflejado en los porcentajes obtenidos para las áreas de cambio $(16.3 \%)$ y permanencias $(83.7 \%)$. Esto es debido a que ya existían extensas áreas intervenidas antes del período de estudio, localizadas principalmente en la zona sur del lago de Maracaibo y en la costa occidental y oriental de la región; de esta manera estas áreas se manifestaron como áreas estables entre el período considerado (Huber, 2007; Bevilacqua et al., 2001).

\section{Discusión de resultados}

Los mapas de vegetación (mapas de cobertura de la tierra o land cover map en inglés) son los más adecuados para mostrar la distribución espacial de la vegetación en un determinado momento. Por esta razón los mapas se encuentran referidos a una extensión de terreno determinada y es importante emplear una misma escala horizontal que permita la comparación entre las diferentes unidades allí presentes (Huber y Oliveira-Miranda. 2010). En este caso los mapas utilizados representaron un acierto al ser producidos en la misma escala; sin embargo, los criterios utilizados para la elaboración de ambos, así como las leyendas fueron diferentes, lo cual se resolvió realizando el proceso de homogenización.

Los resultados obtenidos en esta investigación concuerdan con los reportados para el país en cuanto a la disminución de coberturas naturales principalmente los bosques. Oliveira-Miranda et al. (2010) indican que, a nivel nacional, las áreas intervenidas aumentaron de $102912 \mathrm{~km}^{2}$ a $189147 \mathrm{~km}^{2}$. Señalado incremento significó la eliminación del $9.0 \%$ de las formaciones vegetales del país. Las formaciones con diferentes grados de intervención pasaron de $149493 \mathrm{~km}^{2}$ a $465763 \mathrm{~km}^{2}$, de los cuales $72663 \mathrm{~km}^{2}$ se encuentran, en 2010, en condiciones de alteración o modificación muy alta. La superficie calculada de las áreas intervenidas de esta investigación muestra una diferencia de $19905.0 \mathrm{~km}^{2}$ respecto a la calculada por Oliveira-Miranda et al. (2010), lo que puede ser atribuido a la generalización realizada por categorías para realizar la comparación de los productos cartográficos y la no consideración de las áreas intervenidas localizadas al sur del río Orinoco. Sin embargo, ambos estudios muestran un aumento importante producto de las actividades antrópicas descritas previamente.

De igual forma, Madi et al. (2011) indican que las áreas intervenidas o áreas con cierto grado de intervención han aumentado en detrimento de la vegetación natural del país; los autores indican que en el "Mapa de Vegetación de Venezuela de Huber y Alarcón (1988)" estimaron que las áreas intervenidas cubrían para 1988 un aproximado de $101218 \mathrm{~km}^{2}$ (planos) mientras que para 2007, se estimaron en $148860 \mathrm{~km}^{2}$ en la última versión del Mapa de Vegetación de Venezuela denominada "Paisajes Vegetales" que se esperaba publicar en 2007 en el "Atlas de Venezuela" del Instituto Geográfico Venezolano Simón Bolívar (IGVSB). Esta cifra representó un incremento del $47 \%$ en 19 años, valores que representan un 


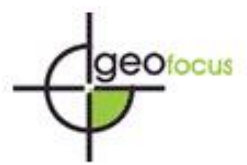

Revista Internacional de Ciencia y Tecnología de la Información Geográfica

International Review of Geographical Information Science and Technology

Morales Montero, O. F., Álbarran Torres, A. J., Gutiérrez Camargo, J. J. (2021). Estudio espacio temporal de la cobertura de la tierra en el norte del río Orinoco-Venezuela (1988-2010). GeoFocus, Revista Internacional de Ciencia y Tecnología de la Información Geográfica, 28, 71-95. http://dx.doi.org/10.21138/GF.686

aumento de esta cobertura entre el $21 \%$ hasta el $31 \%$ en el territorio ubicado al norte del Orinoco (incluyendo el estado Delta Amacuro). De igual manera en el mapa de "Grados de intervención de las formaciones vegetales en Venezuela" elaborado por Madi et al. (2011) indica que los valores de estado de conservación de las formaciones vegetales a nivel nacional, reflejan numerosos hábitats y sistemas ecológicos, poco a nada alterados al sur del Orinoco, mientras que al norte de Orinoco se aprecian la mayor parte de las áreas intervenidas a un grado tal que apenas el $8 \%$ de este territorio se encuentra en grado de intervención "Bueno" gracias a figuras de protección como los sistemas de áreas protegidas o regiones con climas extremos o zonas poco adecuadas para el asentamiento y las actividades humanas económicamente rentables. El señalado territorio es el espacio más modificado del país, y en él los hábitats más afectados son los diferentes tipos de bosques que en muchas y extensas áreas alcanzan un grado de intervención "Pobre; fuertemente intervenido".

Pacheco et al. (2011a) indicaron que entre el período 1920-2008 se perdieron aproximadamente 17935800 ha de bosques venezolanos, siendo las zonas más afectadas la región noroccidental, específicamente los Llanos occidentales y el sur del Lago de Maracaibo, ambas regiones localizadas en la zona norte del río Orinoco. El período entre 1982-1995 ha sido el más crítico para todo el país por presentar la tasa promedio anual de pérdida más alta del período, con 528522 ha, producto de cambios en el uso y ocupación del suelo que desde épocas precolombinas ocasionaros grandes pérdidas de la cobertura vegetal. Para el año 1920 Venezuela poseía una superficie boscosa de 67854900 ha; es decir, $74.0 \%$ de la superficie de país estaba ocupada por bosques, de los que $66.1 \%$ se ubicaban en la región sur y $33.9 \%$ al norte del río Orinoco (23009 596.5 ha); de este último porcentaje $19.9 \%$ pertenece a la región Noroccidental, $5.9 \%$ a la región Centro-Norte y $7.9 \%$ a la región Nororiental. Esta superficie descendió en 2008 a 49919100 ha de las cuales el 26.6\% (13 298448.2 ha) se encuentran al norte del río Orinoco, los datos señalados evidencian las pérdidas de bosques en el país especialmente para la zona norte datos corroborados con los obtenidos en esta investigación donde se señala que los Llanos ha sido la región más afectada en cuanto a la pérdida de formaciones naturales vegetales, especialmente de bosques y sabanas y herbazales.

Por otra parte Vargas y Rosales (2014) señalan que la realidad del país no es nada alentadora en lo que concierne a la conservación y protección de los espacios naturales, pues a pesar de que se cuenta con una amplia normativa legal y un gran número de Área Bajo Régimen de Administración Especial (ABRAE), el problema de la deforestación y los cambios en el uso de la tierra están inmerso en ellas. La principal amenaza ambiental de Venezuela son las deforestaciones. Los bosques del país cubrían 46 millones de hectáreas para el 2010, incluyendo el medio millón de hectáreas de plantaciones de pino en el oriente del país. Desde 1980, cuando los bosques cubrían aproximadamente 58 millones de hectáreas, hasta el 2010 se destruyeron más de 10 millones de hectáreas de bosques naturales, a una tasa promedio de 347000 hectáreas anuales durante 30 años consecutivos siendo los bosques localizados al norte del río Orinoco los más afectados donde se calculó una reducción de $69762.3 \mathrm{~km}^{2}$ de bosques en la zona norte representando la pérdida de casi 7 millones de hectáreas de bosques solo en la zona norte del río Orinoco entre el periodo 1980-2010.

Los resultados obtenidos muestran que los bosques localizados al norte del río Orinoco han reducido la superficie ocupada, de igual forma otras formaciones vegetales tales como las sabanas y herbazales, los matorrales y los matorrales espinosos muestran disminución, en el informe publicado por la FAO y Cegarra et al. (2015) para Venezuela señalan que la extensión de bosques del país para el año 2015 fue de $466830 \mathrm{~km}^{2}$, y la pérdida de los mismos por deforestación entre el periodo 2010-2015 fue de 
Morales Montero, O. F., Álbarran Torres, A. J., Gutiérrez Camargo, J. J. (2021). Estudio espacio temporal de la cobertura de la tierra en el norte del río Orinoco-Venezuela (1988-2010). GeoFocus, Revista Internacional de Ciencia y Tecnología de la Información Geográfica, 28, 71-95. http://dx.doi.org/10.21138/GF.686

164400 ha/año que equivale a una tasa media anual de $0.3 \%$; para 1990 la superficie de bosques era de 52026000 ha; para 1995 a nivel nacional la superficie de los bosques era de 50588801 ha; para el año 2000 la superficie era de 49151000 ha y finalmente para el año 2010 fue de 47505373 ha, registrando una pérdida de 4520627 ha entre el período 1990-2010 siendo los bosques localizados al norte del río Orinoco los más afectados, lo cual es congruente con las tendencias observadas en otros estudios.

Huber (2007) afirma que de continuar las actuales tendencias de crecimiento demográfico y de ocupación poco planificada y ordenada del espacio durante las próximas décadas, se puede vislumbrar una fuerte disminución de las áreas con vegetación natural en casi todos los paisajes vegetales ubicados al norte del Orinoco, hecho que se puede corroborar con los datos obtenidos en esta investigación donde se observa una reducción de las formaciones vegetales presentes en la zona norte del río Orinoco siendo los más afectados la categoría de bosques y sabanas y herbazales. De igual manera Oliveira-Miranda et al. (2010) señalan la disminución de casi todas formaciones vegetales presentes en el país y fundamentalmente las localizadas al norte. Los ecosistemas más notables, tales como las selvas nubladas, las sabanas inundables con morichales, los páramos, y los cardonales sobrevivirán esencialmente. Por el resto, se puede asumir que las comunidades secundarias, tales como el rastrojo herbáceo y el matorral arbustivo constituidos por especies de plantas mayormente cosmopolitas o de amplia distribución tropical, formarán el paisaje vegetal más extenso al norte del Orinoco.

\section{Conclusiones}

Para el periodo analizado, la cobertura bosques fue la que perdió más superficie pasando de $176952.2 \mathrm{~km}^{2}$ para el año 1988, lo que representaba un $37 \%$ del área total para la zona, a $107189.9 \mathrm{~km}^{2}$ para el año 2010, lo que representaba solo el $22 \%$, identificándose una reducción de $69762 . \mathrm{km}^{2}$. En cambio, las áreas intervenidas constituyen la cobertura que más aumentó: para el año 1988 ocupaban $105375.01 \mathrm{~km}^{2}$, lo que representaba un $21 \%$ de área total de la zona, para el año 2010 pasaron a ocupar $209052.01 \mathrm{~km}^{2}$, lo que representó un $43 \%$, identificándose un aumento de $103677.00 \mathrm{~km}^{2}$. Por otra parte, las coberturas de sabanas y herbazales, matorrales espinosos, matorrales, manglares y vegetación de páramo presentaron una reducción en su superficie, a diferencia de la cobertura de cuerpos de agua que mostro un incremento en su superficie ocupada en el periodo de estudio.

Las principales causas del detrimento de la vegetación natural de la zona norte del río Orinoco y fundamentalmente de las coberturas de bosques, sabanas-herbazales y matorrales fueron las actividades antropogénicas que se han desarrollado antes y durante del periodo de estudio (1988-2010), destacando como principales causas la deforestación, explotación forestal, expansión de la frontera agrícola y agropecuaria, aumento sostenido de la población y expansión de áreas urbanas y suburbanas.

Por su parte, la matriz de cambio obtenida permitió observar la invariabilidad espacial de las categorías seleccionadas dentro del periodo de estudio (1988-2010), siendo las coberturas: cuerpos de agua, manglares y vegetación de páramo las que mantuvieron poca variabilidad espacial durante el periodo analizado. Por su parte, experimentaron una importante variabilidad espacial las coberturas: áreas intervenidas, bosques, sabanas y herbazales, matorrales y matorrales espinosos. La direccionalidad de los cambios indicó que todas las coberturas vegetales de la zona, principalmente los bosques, sabanasherbazales y matorrales, cedieron superficie a favor de las áreas intervenidas 


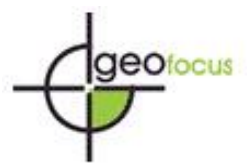

Morales Montero, O. F., Álbarran Torres, A. J., Gutiérrez Camargo, J. J. (2021). Estudio espacio temporal de la cobertura de la tierra en el norte del río Orinoco-Venezuela (1988-2010). GeoFocus, Revista Internacional de Ciencia y Tecnología de la Información Geográfica, 28, 71-95. http://dx.doi.org/10.21138/GF.686

El análisis del mapa de cambios y permanencias muestra que la región natural del delta del Orinoco fue la que mantuvo mayor estabilidad espacial, con un porcentaje superior al $85.0 \%$ de su superficie, correspondiéndose a la realidad físico-geográfica y, en parte por las figuras legales de protección presentes en la región. Las demás regiones que conforman la zona mostraron mayor dinamismo, presentando menos de $85.0 \%$ de estabilidad de superficie, siendo la región de los Llanos la que mostró mayor variabilidad espacial, ya que solo mantuvo estable el $68.7 \%$ de su superficie, indicando de esta forma la fuerte intervención a la que ha sido sometida esta región.

Considerando lo expuesto anteriormente, el comportamiento de los cambios de la cobertura de la tierra de la zona es originado principalmente por actividades antropogénicas. En consecuencia, la vegetación natural de la zona para el periodo 1988-2010 se ve disminuida, lo que ha ocasionado la transformación e incluso la desaparición de la cobertura vegetal natural a vegetación secundaria o a espacios totalmente intervenidos. En consecuencia, es de suma importancia que se implementen nuevas estrategias para la conservación y protección de las distintas formaciones vegetales descritas en esta investigación, siendo una de las principales estrategias la designación de distintas áreas protegidas como las ABRAE, las cuales permiten proteger los ecosistemas y aprovechar de manera sostenible la biodiversidad que ellos albergan y resguardar sus suelos y aguas. A pesar de ello es importante señalar que el hecho de que un área protegida haya sido designada, no garantiza que sus especies y ecosistemas estén bien resguardados; no obstante, existe la evidencia que el status de los ecosistemas tiende a ser mejor dentro de áreas protegidas que fuera de estas.

\section{Referencias bibliográficas}

Albarrán, A. (2008). “Análisis del modelo hipertemporal de la cobertura vegetal de Venezuela en el periodo 1998-2005". (Tesis de pregrado) Universidad de los Andes. Mérida- Venezuela.

Aldana, A., Bosque Sendra, J. (2008). "Cambios ocurridos en la cobertura/uso de la tierra del parque nacional Sierra de la Culata. Mérida-Venezuela. Período 1988-2003”, GeoFocus, 8, pp. 139-168. ISSN: 1578-5157.

Andressen, R. (2007). "Circulación atmosférica y tipos de climas”, en Pantin, G., Reyes, A., Montero, R., Cunill Grau, P., Goyo, G., Márquez, A., Sotillo, A., Aizpúrua, J., Fontanillas, G. (Eds.): Geo Venezuela. Medio físico y recursos ambientales, Caracas-Venezuela, Fundación Empresas Polar Tomo 2, pp. 238329.

Arismendi, J. (2007a). "Presentación geográfica de las formas del relieve", en Pantin, G., Reyes, A., Montero, R., Cunill Grau, P., Goyo, G., Márquez, A., Sotillo, A., Aizpúrua, J y Fontanillas, G. (Eds.): Geo Venezuela. Medio físico y recursos ambientales, Caracas-Venezuela, Fundación Empresas Polar Tomo 2, pp. 128-182.

Arismendi, J. (2007b). Mapa de unidades físico naturales de la República Bolivariana de Venezuela, escala 1: 2500 000. Caracas-Venezuela. Fundación Empresas Polar, Apéndice Cartográfico, Geo Venezuela. Caja A. MN004.

Armenteras, D., González, T. (2016). "Degradación de bosques: contexto y definiciones", en Armenteras, D., González, T., Retana, J y Espelta, J. (Eds.): Degradación de bosques en Latinoamérica: Síntesis 


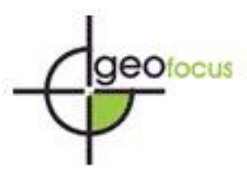

Morales Montero, O. F., Álbarran Torres, A. J., Gutiérrez Camargo, J. J. (2021). Estudio espacio temporal de la cobertura de la tierra en el norte del río Orinoco-Venezuela (1988-2010). GeoFocus, Revista Internacional de Ciencia y Tecnología de la Información Geográfica, 28, 71-95. http://dx.doi.org/10.21138/GF.686

conceptual, metodologías de evaluación y casos de estudio nacionales, publicado por IBERO-REDD+, pp. 9-11.

Armenteras, D. y Rodríguez, N. (2014). "Dinámicas y causas de deforestación en bosques de Latino América: Una revisión desde 1990", Colombia Forestal, N², Vol 17, pp. 233-246. DOI: $\underline{\text { https://doi.org/10.14483/udistrital.jour.colomb.for.2014.2.a07. }}$

Aymard, G. (2011). "Bosques húmedos macrotérmicos de Venezuela", Biollania, edición especial N¹0, pp. 33-46. ISBN 980-231-131-6.

Aymard, G., Farreras, J., Schargel, R. (2011). "Bosques secos macrotérmicos de Venezuela", Biollania, edición especial $\mathrm{N}^{\circ} 10$, pp. 155-177. ISBN 980-231-131-6.

Azócar, A., Fariñas, M. (2003). "Páramos", en Aguilera, M., Azócar, A y Gonzáles, E. (Eds.): Tomo II. Biodiversidad de Venezuela, Caracas-Venezuela, Fundación Polar, Ministerio de Ciencia y Tecnología, Fondo Nacional para la Ciencia, Tecnología e Innovación (FONACIT). Editorial ExLibris, pp. 716-733.

Bevilacqua, M., Cárdenas, L., Liz, A., Hernández, L., Lares, E., Mansutti, A., Miranda, M., Ochoa, J., Rodríguez, M y Solig, E. (2002). Situación de los bosques de Venezuela. La región de Guayana como caso de estudio. Caracas-Venezuela. Global Forest Watch y Fundación Polar.

Cárdenas, A., Carpio, R y Escamilla, F. (2000). Geografía de Venezuela. Caracas-Venezuela. Editora FEDUPEL.

Centeno, J. (2016). "La deforestación en Venezuela". ECODESARROLLO, pp. 3-16.

Chuvieco, E. (1998). "El factor temporal en teledetección: Evolución fenomenológica y análisis de cambios", Revista de Teledetección, numero ${ }^{\circ} 10$, pp. 1-9.

Chuvieco, E. (1995). Fundamentos de teledetección. Madrid-España. Ediciones Rialp, segunda edición, pp 449.

Conde, J., Carlos, C. (2003). "Ecosistemas marino-costeros”, en Aguilera, M., Azócar, A y Gonzáles, E. (Eds.): Tomo II. Biodiversidad de Venezuela, Caracas-Venezuela, Fundación Polar, Ministerio de Ciencia y Tecnología, Fondo Nacional para la Ciencia, Tecnología e Innovación (FONACIT). Editorial ExLibris, pp. 862-883.

Gómez H., Molina, M. (2007). "Principales causas de la deforestación en la vertiente sur del parque nacional Sierra Nevada-Venezuela", Revista Forestal Venezolana, 51, 1, pp. 25-34.

Graces, J., Gutiérrez, A., Salas, R. (S/F). Obras hidráulicas, canales, sistemas de riego y embalses. Programa: Historia de la Ingeniería en Venezuela. Proyecto: Historia de la Ingeniería Estructural de Venezuela.

Hernández, E., Pozzobon, E. (2002). "Tasas de deforestación en cuatro cuencas montañosas del occidente de Venezuela", Revista Forestal Venezolana, 46, 1, pp. 35-42.

Huber, O. (2007). "Los grandes paisajes vegetales", en Pantin, G., Reyes, A., Montero, R., Cunill Grau, P., Goyo, G., Márquez, A., Sotillo, A., Aizpúrua, J y Fontanillas, G. (Eds.): Geo Venezuela. Medio físico y recursos ambientales, Caracas-Venezuela, Fundación Empresas Polar Tomo 2, pp. 538-575. 


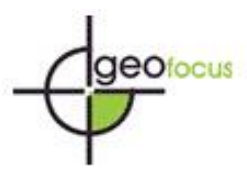

Morales Montero, O. F., Álbarran Torres, A. J., Gutiérrez Camargo, J. J. (2021). Estudio espacio temporal de la cobertura de la tierra en el norte del río Orinoco-Venezuela (1988-2010). GeoFocus, Revista Internacional de Ciencia y Tecnología de la Información Geográfica, 28, 71-95. http://dx.doi.org/10.21138/GF.686

Huber, O., Alarcón, C. (1988). Mapa de Vegetación de Venezuela, 1:2.000.000. Caracas-Venezuela. MARNR-BIOMA.

Huber, O., Oliveira-Miranda, M. (2010). “Ambientes terrestres de Venezuela”, en Rodríguez, J., RojasSuárez, F. y Giraldo, D. (Eds.): Libro Rojo de los Ecosistemas Terrestres de Venezuela. CaracasVenezuela, Provita. Shell Venezuela. Lenovo (Venezuela), pp. 26-86.

Instituto Geográfico de Venezuela "Simón Bolívar". (IGVSB). (2014). Atlas de la República Bolivariana de Venezuela. Caracas-Venezuela.

Instituto Venezolano de Investigaciones Científicas (IVIC). Centro Internacional de Ecología Tropical (CIET). Unesco Rodríguez, J., Lazo, R., Solórzano, L y Rojas-Suárez, F. (1998). Venezuela digital: Mapa de vegetación. Caracas-Venezuela. Disponible en http://ecosig.ivic.ve.

José de Jesús, S., Montes, R. (2006). "Diversidad y conservación de las sabanas llaneras", en Duno de Stefano, R., Aymard, G y Huber, O. (Ed.): Flora vascular de los llanos de Venezuela. Caracas-Venezuela, FUDENA, Fundación Empresas Polar, FIBV, pp. 87-90.

León, A., Madi, Y., Vázquez, J., Rodrigues, J., Duarte, E y Uzcátegui, J. (2011). Grados de intervención de las formaciones vegetales en parques nacionales de Venezuela. Caso de estudio: Henri Pittier, Yacambú y San Camilo. La Habana-Cuba. Trabajo presentado en el VIII Congreso sobre Áreas Protegidas de la VIII Convención Internacional sobre Medio Ambiente y Desarrollo.

Madi, Y., Vázquez, J., León, A., Rodríguez, J. (2011). "Estado de conservación de los bosques y otras formaciones vegetales en Venezuela", Biollania, edición especial N 10 , pp. 302-324. ISBN 980-231-1316.

Maldonado, H., Parra, A y Aldana, A. (2011). "La deforestación en la reserva forestal Caparo-Venezuela períodos 1987-1994, 1994-2007 y 1987-2007. (Aplicación de la teledetección y los SIG)", Revista Forestal Latinoamericana, 26, 2, pp. 107-132.

Martínez, B., Gilabert, M., del Barrio, G y Melía, J. (2001). "Desarrollo de una metodología de detección de cambios en zonas susceptibles de procesos de degradación", en Rossell, J y Martínez, J. (Eds.): Teledetección, Medio Ambiente y Cambio Global. Cataluña-España, Milenio Publicaciones S.L, pp. 110113.

Mateucci, S., Colma, A. (1997). "Agricultura sostenible y ecosistemas áridos y semiáridos de Venezuela”, INTERCIENCIA, $\mathrm{n}^{\circ}$ 3, volumen 22, pp. 123-130. ISNN: 0378-1844.

Ministerio del Ambiente y de los Recursos Naturales (MARNR). (2006). Los manglares venezolanos. Caracas-Venezuela. Proyecto: Planificación y Ordenamiento de los ecosistemas de manglares para el manejo sostenible con la participación de las comunidades indígenas del delta del Orinoco.

Molina, Z. y Albarrán, A. (2013). "Análisis multitemporal y de la estructura horizontal de la cobertura de la tierra: parque nacional Yacambú, estado Lara-Venezuela”, Cuadernos de Geografía: Revista Colombiana de Geografía, número 1, volumen $2, \quad$ pp. 25-40. DOI: https://doi.org/10.15446/rcdg.v22n1.36305. 


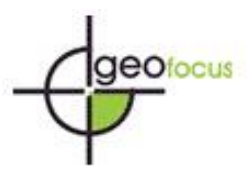

Morales Montero, O. F., Álbarran Torres, A. J., Gutiérrez Camargo, J. J. (2021). Estudio espacio temporal de la cobertura de la tierra en el norte del río Orinoco-Venezuela (1988-2010). GeoFocus, Revista Internacional de Ciencia y Tecnología de la Información Geográfica, 28, 71-95. http://dx.doi.org/10.21138/GF.686

Molina., Z., Arias, M., Mejía, J. (2009). "Transformaciones espaciales en las microcuencas la Cuesta-La Boba estado Mérida-Venezuela. Período 1952-2004”, Revista Geográfica Venezolana, número 1, volumen 50, pp. 109-129.

Molinillo, M., Monasterio, M. (2002). "Patrones de vegetación y pastoreo en ambientes de páramo", ECOTRÓPICOS-Sociedad Venezolana de Ecología, número 1, volumen 15, pp. 19-34.

Morales-Rodríguez, M., Chacón-Moreno, E y Ataroff, M. (2009). "Transformaciones del paisaje de selvas de montaña en la cuenca del río Capaz, Andes Venezolanos”, ECOTRÓPICOS-Sociedad Venezolana de Ecología, número 2, volumen 22, pp. 64-82.

Morales, O., Gutiérrez, J. (2017). "Determinación y análisis de las transformaciones espaciales de la cobertura de la tierra para Venezuela, período 1988-2010. Validación: Microcuenca de la quebrada la Toma, Período 1988-2015”. (Tesis de pregrado) Universidad de los Andes. Mérida- Venezuela.

Moreau, A. (2007). "Las medidas fundamentales". en Pantin, G., Reyes, A., Montero, R., Cunill Grau, P., Goyo, G., Márquez, A., Sotillo, A., Aizpúrua, J y Fontanillas, G. (Eds.): Geo Venezuela. La Geografía histórica del poblamiento territorial venezolano-La tropicalidad venezolana. Caracas-Venezuela, Fundación Empresas Polar Tomo 1, pp. 378-412.

Nassar, J., Velásquez, G., Romero-Briceño, J y Medina, E. (2013). "Las cactáceas como elementos de caracterización de ambientes áridos y semiáridos en Venezuela", en Medina, E., Huber, O., Nassar, J y Navarro, P. (Eds.): Recorriendo el paisaje vegetal de Venezuela. Caracas-Venezuela. Ediciones IVIC, Instituto Venezolano de Investigaciones Científicas (IVIC), pp. 97-124.

Organización de las Naciones Unidas para la Agricultura y Alimentación. (FAO). (2010). Evaluación de los recursos forestales mundiales 2010-informe principal. Roma-Italia. Estudio-FAO: Montes Núm 163.

Organización de las Naciones Unidas para la Agricultura y Alimentación. (FAO). (2011). Situación de los bosques del mundo. Roma-Italia. Organización de las Naciones Unidas para la Agricultura y la Alimentación.

Organización de las Naciones Unidas para la Agricultura y Alimentación. (FAO) y Cegarra, J., Catalán, A., Flores, J y Azuaje, J. 2015. Evaluación de los recursos forestales mundiales 2015-Informe Nacional. Venezuela. Roma-Italia.

Oliveira-Miranda, M., Huber, O., Rodríguez, J., Rojas-Suárez, F., Oliveira-Miranda, R., ZambranoMartínez, S y Giraldo, D. (2010). "Riesgo de eliminación de los ecosistemas terrestres de Venezuela", en Rodríguez, J., Rojas-Suárez, F y Giraldo, D. (Ed.): Libro Rojo de los Ecosistemas Terrestres de Venezuela. Caracas-Venezuela, Provita. Shell Venezuela. Lenovo (Venezuela), pp. 109-231.

Pacheco, C., Aguado, I. y Mollicone, D. (2011a). "Dinámica de la deforestación en Venezuela: Análisis de los cambios a partir de mapas históricos", INTERCIENCIA, n 8 , volumen 36, pp. 566-586.

Pacheco, C., Aguado, I. y Mollicone, D. (2011b). "Las causas de la deforestación en Venezuela: Un estudio retrospectivo", Biollania, edición especial N¹0, pp. 281-292.

Petit C. C. y Lambin, E. (2002). "Impact of data integration technique on historical land-use/land cover change: Comparing historical maps with remote sensing data in the Belgian Ardennes", Landscape Ecology, 17, pp. 117-132. 


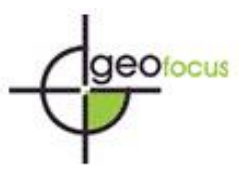

Morales Montero, O. F., Álbarran Torres, A. J., Gutiérrez Camargo, J. J. (2021). Estudio espacio temporal de la cobertura de la tierra en el norte del río Orinoco-Venezuela (1988-2010). GeoFocus, Revista Internacional de Ciencia y Tecnología de la Información Geográfica, 28, 71-95. http://dx.doi.org/10.21138/GF.686

Peñaloza, R., González, J., Guerra, F y Gómez, H. (2008). "La deforestación en la cuenca del río Zulia, Barinas, Venezuela. Análisis preliminar utilizando SIG”, Geoenseñanza, número 1, volumen 13, pp. 7182. ISNN: 1316-6077.

Plonczak, M. (1997). “Tipos de bosque y su presión de uso en Venezuela”, Quebracho, número 6, pp. 6974.

Pozzobon, E., Osorio R. (2002). "Evaluación de las deforestaciones en la reserva forestal de Ticoporo, estado Barinas-Venezuela, en base al análisis multitemporal de imágenes de percepción remota", Revista Geográfica Venezolana, número 2, volumen 43, pp. 215-235.

Pozzobon, E., Hernández, E., Dugarte, I. (2004). "Evaluación del proceso de deforestación en tres cuencas del piedemonte lacustrino de la cordillera de los Andes", Revista Forestal Venezolana, número 2, volumen 48, pp. 14-22.

Rodríguez, P., Rojas-Suárez, F., Giraldo, D. 2010. Libro Rojo de los Ecosistemas Terrestres de Venezuela. Caracas-Venezuela. Provita, Shell Venezuela, Lenovo (Venezuela).

Silva, J. (2003). "Sabanas", en Aguilera, M., Azócar, A y Gonzáles, E. (Eds.): Tomo II. Biodiversidad de Venezuela. Caracas-Venezuela, Fundación Polar, Ministerio de Ciencia y Tecnología, Fondo Nacional para la Ciencia, Tecnología e Innovación (FONACIT). Editorial ExLibris, pp. 678-695.

Soriano, P. y Ruiz, A. (2003). “Arbustales xerófilos”, en Aguilera, M., Azócar, A y Gonzáles, E. (Eds.): Tomo II. Biodiversidad de Venezuela. Caracas-Venezuela, Fundación Polar, Ministerio de Ciencia y Tecnología, Fondo Nacional para la Ciencia, Tecnología e Innovación (FONACIT). Editorial ExLibris, pp. 696-715.

Vargas, F., Chuvieco, E., Salas, J y Meza, E. (2002). "Empleo de la teledetección en el análisis de la deforestación tropical: El caso de la reserva forestal de Ticoporo (Venezuela)", Serie Geográfica, Número 10, pp. 55-76. I.S.S.N: 1136-5277.

Vargas, T., Rosales, C. (2014). "Pérdida de la cobertura vegetal y sus repercusiones ambientales y legales", Geoenseñanza, número 1, volumen 19, pp. 61-79.

Vivas, L. (2012). Geotemas-Venezuela 2012. Mérida-Venezuela. Fundación Fondo Editorial "Simón Rodríguez" de la Lotería del Táchira. 
